\title{
ArcheoSciences
}

Revue d'archéométrie

\section{Grandes phases de pédogenèse, d'érosion et d'anthropisation des sols au cours de la seconde moitié de l'Holocène en Lorraine (France)}

Main phases of soil genesis, erosion and anthropisation during the second half of the Holocene in Lorraine (Eastern France)

\section{Anne Gebhardt, Serge Occhietti et Kai Fechner}

\section{OpenEdition}

Journals

Édition électronique

URL : https://journals.openedition.org/archeosciences/4113

DOI : $10.4000 /$ archeosciences. 4113

ISBN : 978-2-7535-3691-3

ISSN : 2104-3728

Éditeur

Presses universitaires de Rennes

\section{Édition imprimée}

Date de publication : 30 novembre 2014

Pagination : 7-29

ISBN : 978-2-7535-3689-0

ISSN : 1960-1360

Référence électronique

Anne Gebhardt, Serge Occhietti et Kai Fechner, «Grandes phases de pédogenèse, d'érosion et d'anthropisation des sols au cours de la seconde moitié de l'Holocène en Lorraine (France) », ArcheoSciences [En ligne], 38 | 2014, mis en ligne le 30 novembre 2016, consulté le 31 janvier 2022. URL : http://journals.openedition.org/archeosciences/4113; DOI : https://doi.org/10.4000/ archeosciences. 4113 


\title{
Grandes phases de pédogenèse, d'érosion et d'anthropisation des sols au cours de la seconde moitié de l'Holocène en Lorraine (France)
}

\author{
Main Phases of Soil Genesis, Erosion and Anthropisation \\ During the Second Half of the Holocene in Lorraine (Eastern France)
}

\author{
Anne Gebhardt-Even ${ }^{a}$, Serge Occhietti ${ }^{b}$ et Kai Fechner ${ }^{c}$
}

\begin{abstract}
Résumé : Cet article présente les résultats les plus significatifs acquis lors du suivi pédo-sédimentaire, incluant l'analyse micromorphologique, de divers sites archéologiques étudiés au cours de la dernière décennie en Lorraine. Il propose une première reconstitution diachronique des grandes phases de pédogenèse, d'érosion et d'anthropisation des sols à l'échelle de la région. L'érosion des sols, qui semble débuter très localement dès l'Âge du Bronze (voire au Néolithique), se généralise au cours de l'Âge du Fer pour se stabiliser ensuite à l'époque romaine et au Moyen Âge. L'ampleur de cette érosion reste, à l'instar des régions voisines, dépendante de facteurs anthropiques et naturels plus ou moins interdépendants, en particulier les mouvements de population et les variations climatiques. Cette étude de sites lorrains contribue à la réflexion globale sur les variabilités environnementales et les mutations sociales au cours de la seconde moitié de l'Holocène en Europe du Nord.
\end{abstract}

\begin{abstract}
This paper presents the most significant results of the last decade from soil, sedimentary and micromorphology analyses of archaeological sites in Lorraine. A tentative diachronic reconstruction of the main pedogenetical, erosional and anthropisation phases observed at the regional scale is proposed. The soil erosion seems to have started locally during the Bronze Age and perhaps Late Neolithic, developed during Iron Age and stabilized during Roman time and Middle Age. The erosion processes are related to intricated human and natural factors, including population and climatic changes, as observed in neighbouring regions. These results from Lorraine contribute to the more global reflexion on the environmental variability and social mutation appearing through the second half of the Holocene in Northern Europe.
\end{abstract}

Mots clés : Archéo-pédologie, érosion des sols, anthropisation des paysages, phases climatiques holocènes, Lorraine, France.

Keywords: Archaeo-pedology, soil erosion, man-made landscape, Holocene climatic phases, Lorraine, France.

a UMR 7362/CNRS, Laboratoire Image, Ville, Environnement, Université de Strasbourg, 1 rue de l'Argonne, 67000 Strasbourg, France; INRAP. (anne. gebhardt-even@inrap.fr)

${ }^{\mathrm{b}}$ LOTERR, 23 boulevard Albert 1'r, Université de Lorraine, 54015 Nancy, France; Département de géographie, UQAM, CP 8888 Centre-ville, Montréal, Québec., H3C 3P8, Canada. (serge.occhietti@gmail.com)

'INRAP, ZI de la Pilaterie, 11, rue des champs, 59650 Villeneuve-d'Ascq, France; UMR 7362/CNRS, Laboratoire Image, Ville, Environnement, Université de Strasbourg. (Kai.Fechner@inrap.fr) 


\section{INTRODUCTION}

Les successions pédo-sédimentaires enregistrent les grandes phases climatiques de l'Holocène et les vagues successives d'anthropisation qui aboutissent aux paysages actuels. Leur étude est maintenant intégrée aux travaux d'archéologie, comme en Belgique et Luxembourg (Langohr, 2001; Fechner et al., sous presse), en Allemagne (Bork et Lang, 2003; Leopold et Völkel, 2006; Dotterweich, 2008; Dotterweich et Dreibrodt, 2011), en Suisse (Braillard et al., 2002) et en France, notamment en Normandie (GermainVallée et Lespez, 2011), en Alsace (Ertlen et al., 2013; Boës et Gebhardt, 2007), en Champagne (Fechner et Slachmuylder, 2009), dans la vallée du Rhône (Berger et Bravard, 2012) et le Nord-Picardie (Deschodt et Salvador, 2006; Deschodt et al., 2012). En Lorraine, en dépit de quelques études pédologiques classiques (Gury, 1973, 1976; Duchaufour, 1976; Bonneau et al., 1978; Jacquin et Florentin, 1988), l'analyse des interactions de l'Homme sur les sols reste limitée à des thématiques très spécifiques comme les relations entre l'histoire de la végétation et la podzolisation dans les Vosges (Guillet, 1972) ou la dynamique des hautes-chaumes du massif vosgien (Goepp et al., 2008). Pour combler en partie cette lacune, nous présentons des données originales acquises lors du suivi pédo-sédimentaire de divers travaux archéologiques, préventifs et programmés, effectués au cours de la dernière décennie en Lorraine et proposons un premier essai de reconstitution des phases d'érosion des sols de cette région au cours de la seconde moitié de l'Holocène.

Après une présentation des grands ensembles géologiques structurant la Lorraine et des méthodes utilisées, l'article aborde la description pédo-sédimentaire d'une quinzaine de profils archéologiques dont les unités caractéristiques révèlent des phases de pédogenèse et d'érosion-sédimentation datées par des témoins archéologiques. L'analyse de ces données permet ensuite de proposer un cadre diachronique de ces grandes phases à l'échelle de la région. La comparaison de ces données lorraines, replacées dans le cadre plus large des variations climatiques de l'Holocène, avec celles de régions limitrophes, conduit à une réflexion sur la relation entre le forçage climatique et les activités anthropiques à l'origine des grandes phases érosives locales.

\section{ConteXte RÉgional NATUREL}

La Lorraine (fig. 1) comprend la partie orientale du bassin de Paris adossée aux Vosges. Le Massif vosgien inclut un cœur cristallin, le bassin permien de Saint-Dié-des-Vosges et une bordure gréseuse du Trias (grès du Buntsandstein) qui se prolonge jusqu'aux Vosges du Nord. La couverture mésozoïque donne à la Lorraine son paysage caractéristique de côtes calcaires orientées nord/sud, structurant le réseau hydrographique principal (Moselle, Meurthe et Sarre) qui s'écoule vers le nord (Lexa-Chomard et Pautrot, 2006; Le Roux, 2007).

Les formations superficielles lorraines sont composées essentiellement de dépôts associés aux glaciations quaternaires vosgiennes (Flageollet, 2002) et de formations alluviales disposées sur des terrasses ou en fond de vallée (Carcaud, 1992; Cordier et al., 2004). Des limons quaternaires peu épais, discontinus et souvent remaniés, couvrent une partie des plateaux du nord de la Lorraine. Grèzes litées et éboulis de versant sont fréquents, respectivement au pied des versants calcaires du plateau lorrain (Lucas-Leclin, 1992) et sur les pentes des Vosges cristallines (Decaulne, 2007).

Le climat lorrain est tempéré légèrement continental avec des disparités notoires liées au relief. La pluviométrie s'échelonne entre $750 \mathrm{~mm} / \mathrm{an}$ le long du sillon lorrain (vallée moyenne de la Moselle) et $2400 \mathrm{~mm} / \mathrm{an}$ au Ballon d'Alsace (Wahl, 2007).

\section{APPROCHE PÉdo-SÉdIMENTAIRE}

Le profil de référence correspondant au secteur le plus représentatif de chaque site est choisi d'après l'observation des sondages profonds et des bermes témoins laissées dans l'emprise archéologique à l'issue de larges décapages. Chaque profil pédo-sédimentaire inclut des sols plus ou moins érodés enfouis sous des accumulations colluvio-alluviales de fond de vallée ou des colluvions de versant. Dans les vallons, on observe souvent des profils inversés, caractérisés par des horizons organiques sombres et épais, recouverts par des formations limoneuses plus claires issues d'horizons minéraux plus anciens démantelés en amont. Les structures anthropiques (chemins creux, mares, fossés, foyers, trous de poteau...) piègent localement des colluvions accumulées postérieurement à leur usage. Sur les berges, les apports alluviaux de débordement se mêlent parfois aux colluvions et complexifient la lecture des profils.

Pour la description (tableau 1) et l'interprétation (tableau 2) de ces profils, une approche litho-pédostratigraphique est appliquée. Chaque profil (fig. 2) est composé d'une succession d'unités pédologiques (horizons) et/ou sédimentaires (colluvions, alluvions) $[\mathrm{U}]$ analysées selon les critères pédo-sédimentologiques (granulométrie, macro- et microstructures). À partir de ces descriptions, les unités sont interprétées et regroupées en séquences génétiques [S] correspondant à des phases dynamiques homogènes de sédimen- 


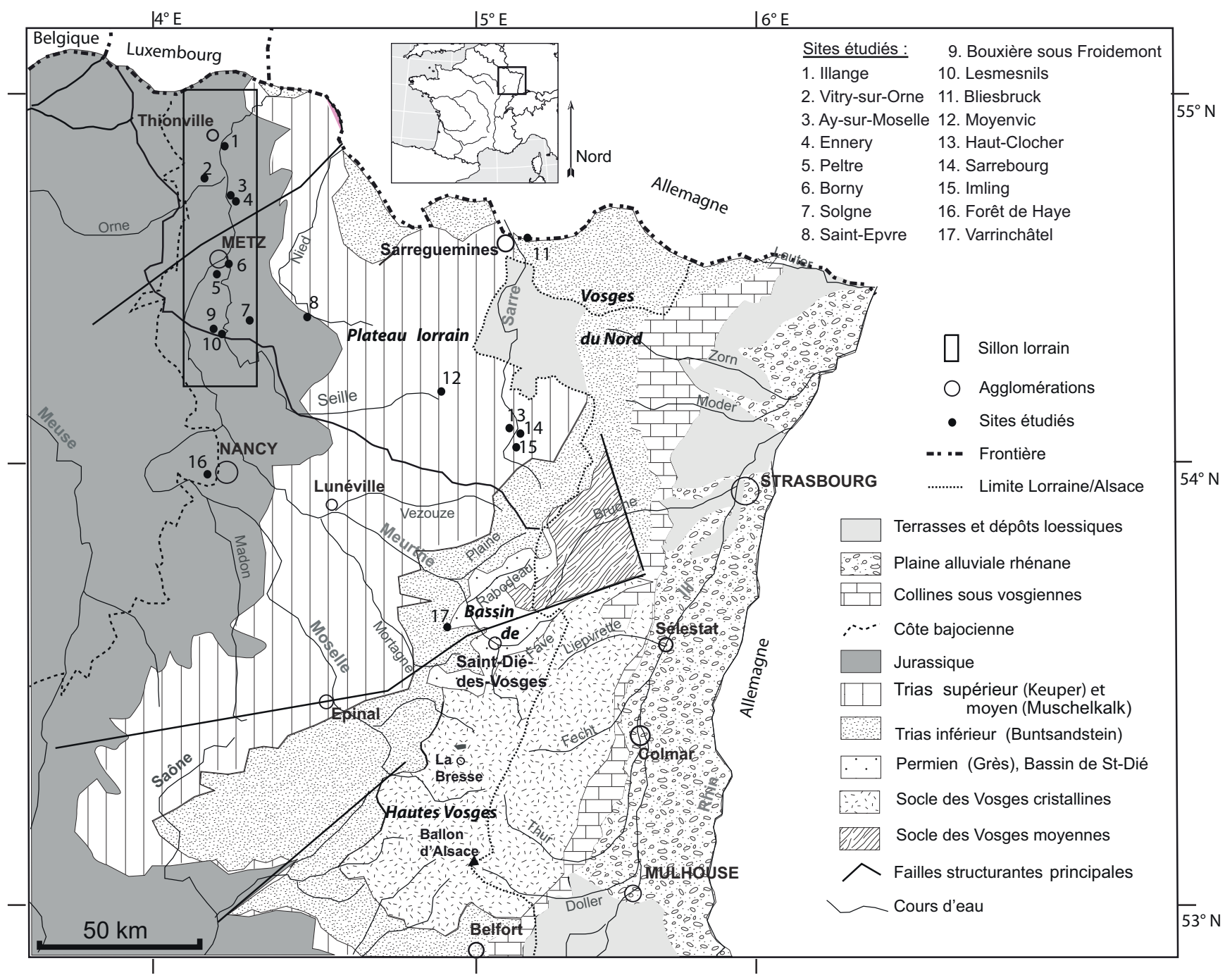

Figure 1 : Localisation des sites étudiés, sur la carte géologique simplifiée de la région, d'après Lexa-Chomard et Pautrot (2006) et Sell et al. (1998). Les Vosges cristallines correspondent au socle, la Lorraine gréseuse correspond au grès permien et au Trias inférieur et le Plateau lorrain correspond au Trias moyen et supérieur.

Figure 1: Location of the studied sites, on the geological map of the area (simplified from Lexa-Chomard \& Pautrot [2006] and Sell et al. [1998]). The Crystalline Vosges correspond to the basement, the "Lorraine gréseuse" to the Permian and lower Trias sandstones, the "Plateau lorrain" to the middle and upper Trias, respectively.

tation ou de pédogenèse. Les limites de séquence sont soit des contacts d'érosion, soit des variations granulométriques et microstructurales correspondant à des changements de dynamique sédimentaire ou pédologique. Les horizons pédologiques sont décrits selon les critères macroscopiques classiques de couleur, texture et structure (Duchaufour, 1976; Baize et Jabiol, 1995). La dénomination des sols est celle du référentiel pédologique des principaux sols d'Europe (Baize, 1992). La granulométrie (Laboratoire EOST, Université de Strasbourg) et la pédochimie (Laboratoire d'étude des sols, INRA d'Arras) ont respectivement permis de préciser l'origine alluviale ou colluviale des apports en fond de vallée et de caractériser certains horizons pédologiques. Les observations de terrain ont parfois été prolongées par l'analyse micromorphologique des sols et des sédiments au microscope. Les lames minces $(9 \times 6 \mathrm{~cm})$, réalisées par le Laboratoire Thomas Beckmann (Allemagne), ont été décrites selon la nomenclature internationale (Bullock et al., 1985), traduite en français par Stoops (1986). 


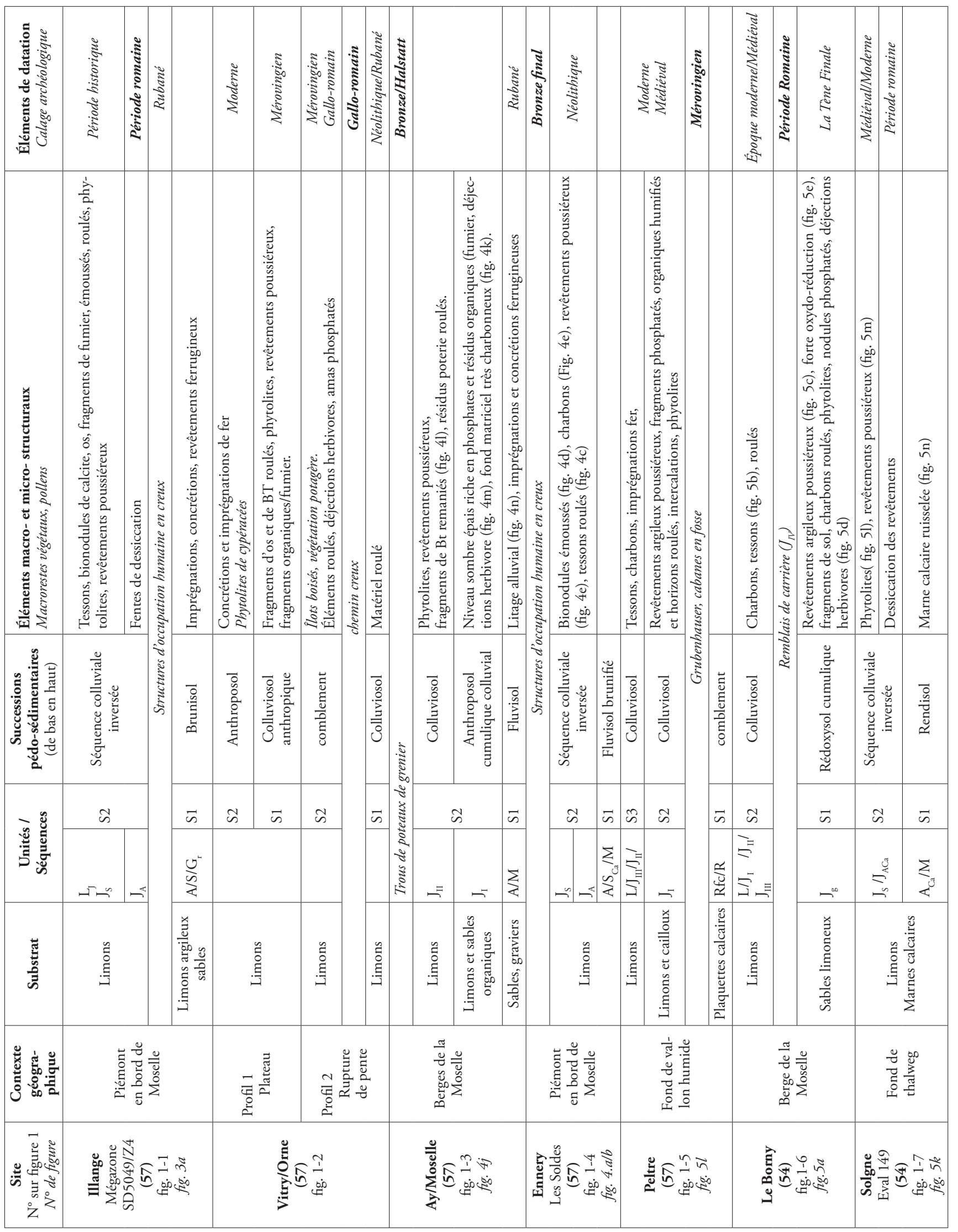




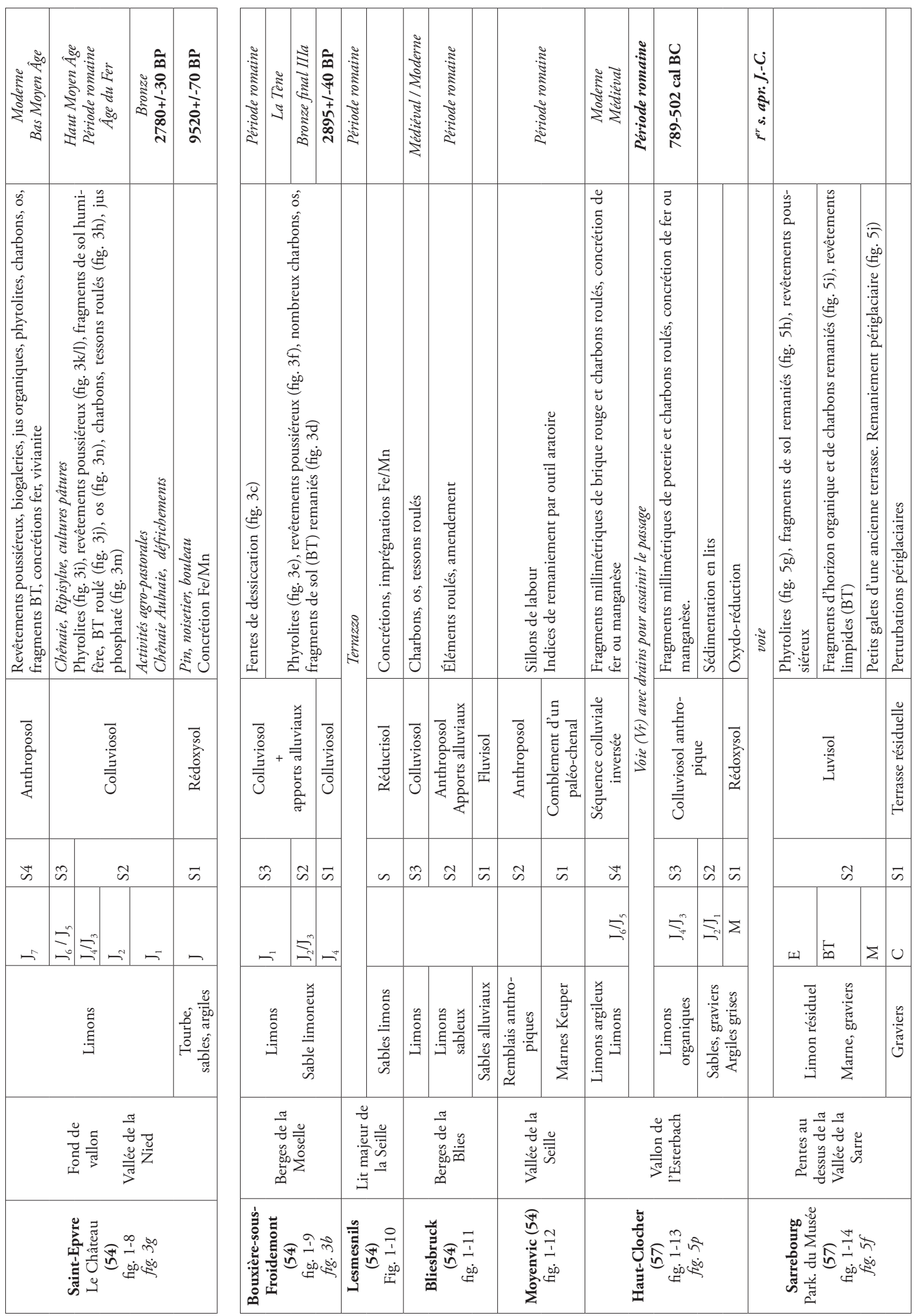




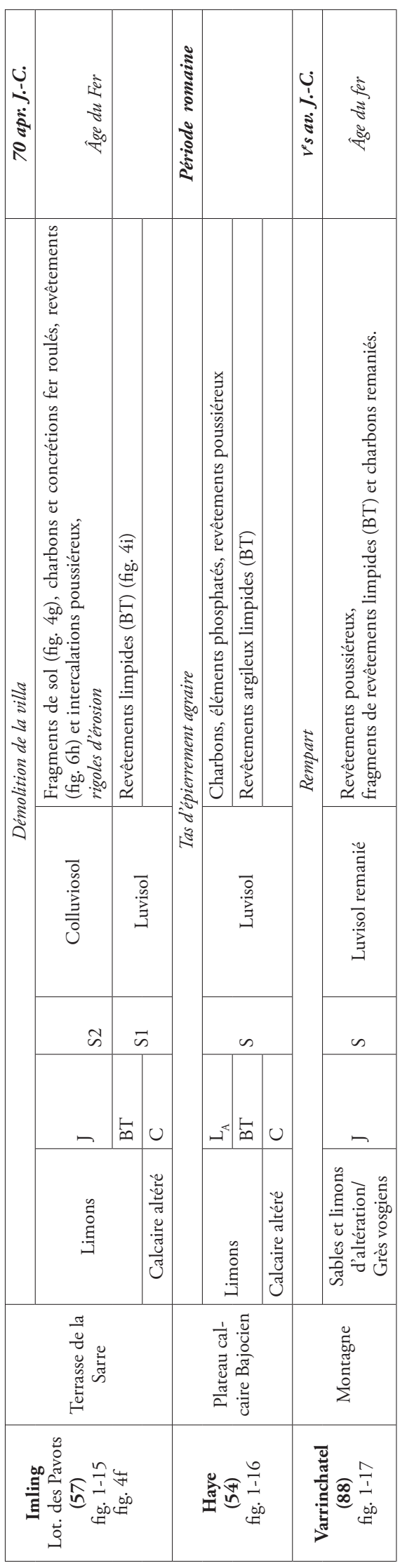

Grâce à de nombreuses recherches en France septentrionale, effectuées en contexte archéologique (Courty et al., 1989; Macphail et al., 1990; Gebhardt, 1993, 2000), expérimental (Gebhardt, 1995, 1999) ou ethnographique (Gebhardt, 2007a), nous possédons un référentiel conséquent de traits micromorphologiques applicable à l'étude de l'anthropisation des paysages lorrains. Ce référentiel documente l'ouverture du milieu, la déforestation, l'érosion, le colluvionnement, les labours, le parcage/pâturage et les variations des niveaux phréatiques.

Le matériel archéologique remanié, inclus dans les dépôts, et plus rarement associé à des occupations en place, apporte un précieux calage chronologique des profils, parfois complété par des datations ${ }^{14} \mathrm{C}$. Pour les âges ${ }^{14} \mathrm{C}$ publiés par d'autres auteurs que nous reprenons ici, nous proposons des équivalences $\mathrm{BP} / \mathrm{cal} \mathrm{BC}$, en petits caractères, estimées avec une probabilité de 2 sigma selon le logiciel Calib 7.0 (Stuiver et Reimer, 1993), afin de permettre les comparaisons. Plusieurs courbes, établies à partir de différents marqueurs climatiques (fig. 6), servent de cadre de référence pour tenter de caler chronologiquement les séquences lorraines, de cerner les interactions entre activités agro-pastorales anciennes et milieu naturel (Gebhardt, 2000) et de pointer les périodes de dégradation et d'instabilité du paysage au cours du temps (Germain-Vallée et Lespez, 2011).

\section{Résultats}

Détaillés dans différents rapports de fouille ou de diagnostic archéologiques et articles, les résultats sont synthétisés sous la forme de deux tableaux (tableaux 1 et 2).

La plupart des sites mentionnés (fig. 1, tableaux 1 et 2) est issue de l'archéologie préventive. Ils sont localisés au nord de la Lorraine, le long des vallées de la Moselle, de la Seille et de la Sarre, soit dans les fonds de vallée, soit sur les versants. Dans les fonds de vallée, on observe un mélange d'apports colluviaux et de dépôts alluviaux. Sur les pentes, le substrat argilo-calcaire peut être recouvert de formations limoneuses d'épaisseur variable plus ou moins remaniées ou érodées, parfois pédogenéisées. Seuls deux sites concernent des recherches archéologiques programmées : l'un en Forêt de Haye, sur le plateau calcaire bajocien et l'autre en contexte plus montagneux, installé sur une butte témoin gréseuse dominant la haute Meurthe au sud-ouest du bassin de Saint-Dié-des-Vosges.

Pour chaque site, le regroupement séquentiel des unités descriptives a révélé une succession pédo-sédimentaire (tableaux 1 et 2) comprenant un sol ancien, plus ou moins érodé voire remanié, enfoui sous une séquence colluviale. 


\begin{tabular}{|c|c|c|c|c|c|c|c|c|c|c|c|c|c|c|c|c|c|c|c|c|c|}
\hline 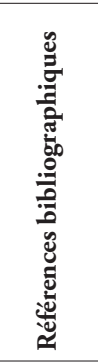 & 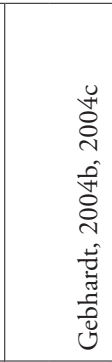 & & & & & 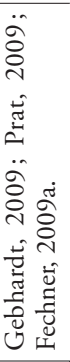 & & & & 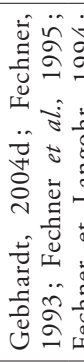 & 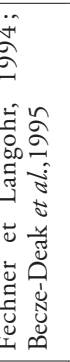 & & & & & 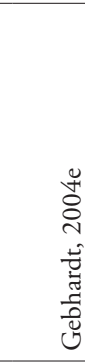 & & & 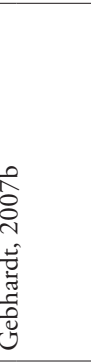 & & \\
\hline 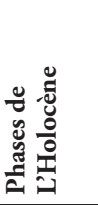 & 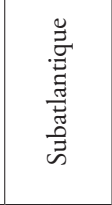 & 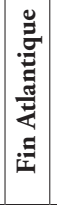 & 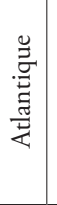 & & 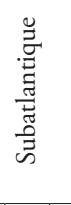 & & 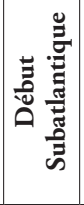 & & 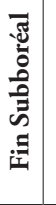 & 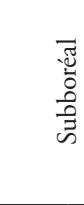 & & $\begin{array}{l}\text { 总 } \\
\text { 总 } \\
\text { 妾 }\end{array}$ & 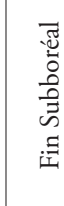 & & & 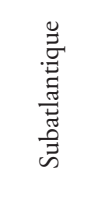 & & & 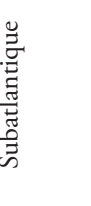 & 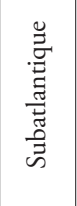 & \\
\hline 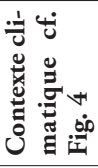 & 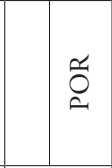 & & & 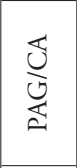 & & 颉 & & & $\overbrace{0}^{q}$ & & & & జे & & 岕 & & & & 岕 & $\underset{0}{0}$ & \\
\hline 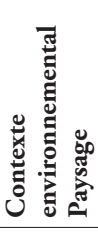 & 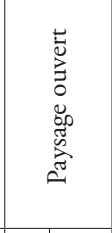 & & & & 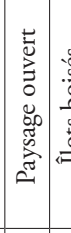 & 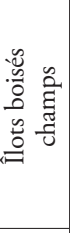 & & & & 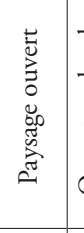 & 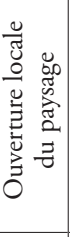 & & & & & 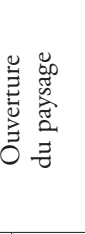 & & & 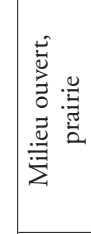 & 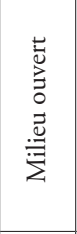 & \\
\hline 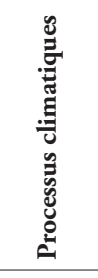 & 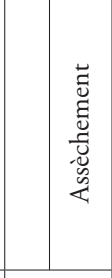 & 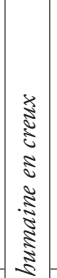 & 菊 & 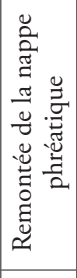 & & & ב气 & & & & & 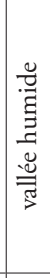 & 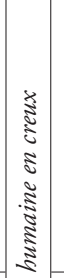 & 总 & 总 & & 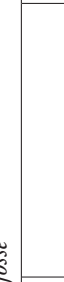 & & 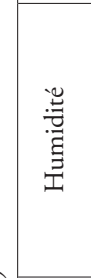 & 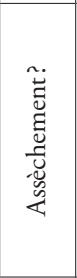 & \\
\hline 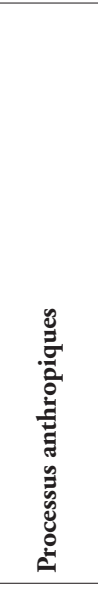 & 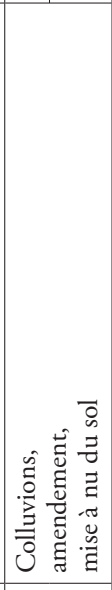 & 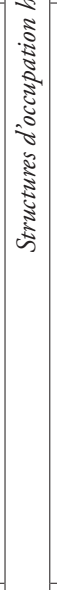 & & 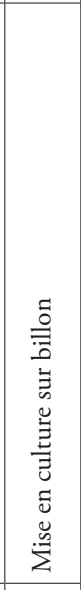 & 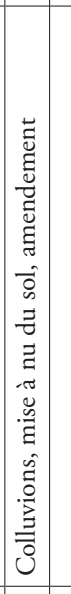 & 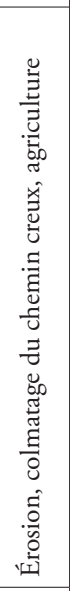 & 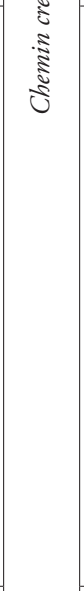 & 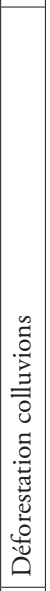 & 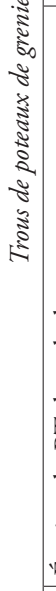 & 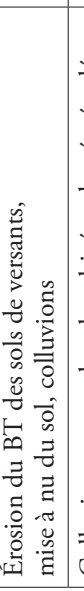 & 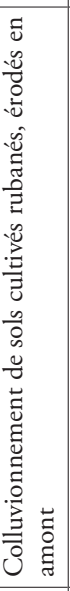 & & 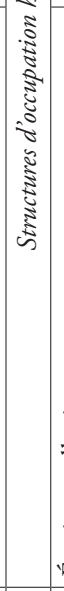 & & 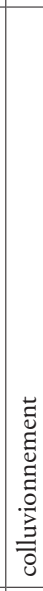 & 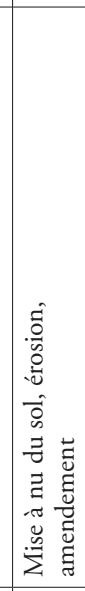 & & 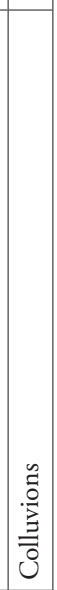 & 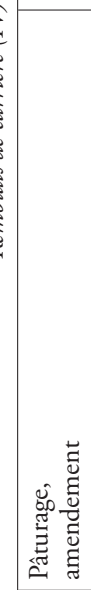 & 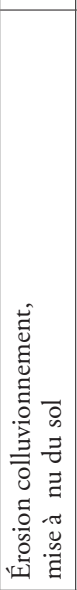 & \\
\hline 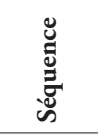 & $\tilde{\omega}$ & & के & $\tilde{\omega}$ & $\bar{n} 0$ & ๙ & & $\vec{n}$ & & $\approx$ & & $\bar{\omega}$ & & $\bar{\omega}$ & $\tilde{n}$ & $\approx$ & जे & $\approx$ & $\bar{n}$ & ஸे & $\bar{n}$ \\
\hline 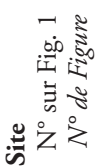 & \multicolumn{3}{|c|}{ 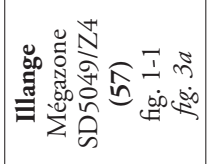 } & \multicolumn{5}{|c|}{ 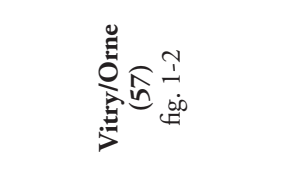 } & \multicolumn{4}{|c|}{ 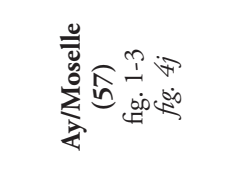 } & \multicolumn{2}{|c|}{ 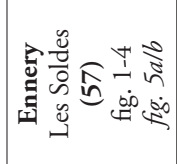 } & \multicolumn{3}{|c|}{ 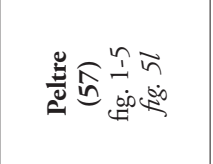 } & \multicolumn{2}{|c|}{ 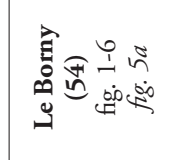 } & \multicolumn{2}{|c|}{ 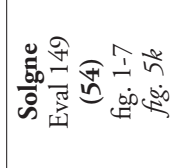 } \\
\hline
\end{tabular}




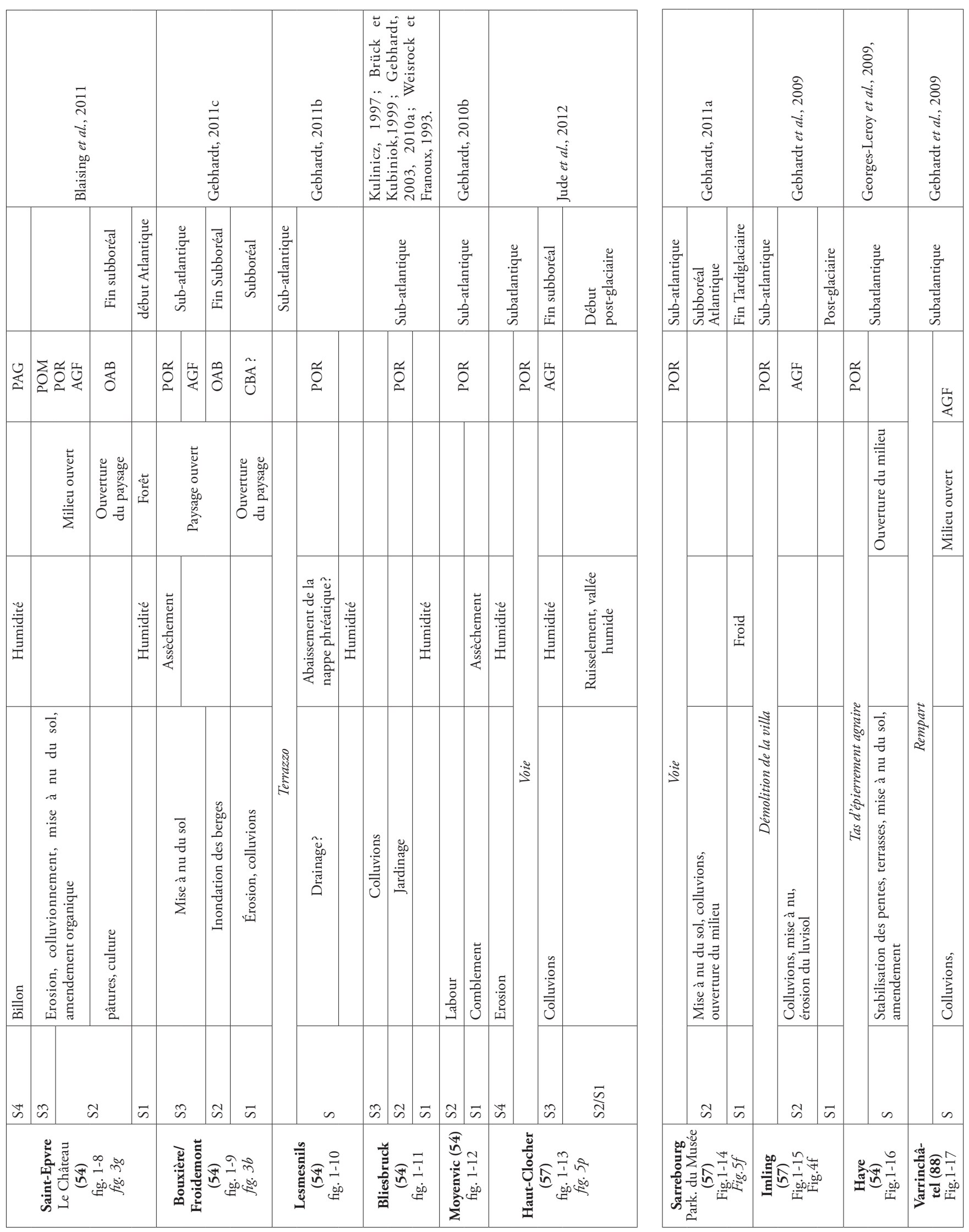


Tableau 2 : (Page précédente) Tableau replaçant les différents processus déduits des observations pédo-sédimentaires dans le contexte climatique (voir légende fig. 6) et environnemental global des grandes phases de l'Holocène. Les lignes brisées représentent des discordances visibles sur le terrain. La dernière colonne fait référence à la bibliographie disponible pour chaque site.

Tableau 2: (Previous page) Table of the erosional and depositional processes inferred from the pedo-sedimentary data and in relation with the climatic and paleoenvironnemental phases of the Holocene (see fig. 6 legend). The broken line refers to a discordance seen in the field. Available references about each site in the last column.

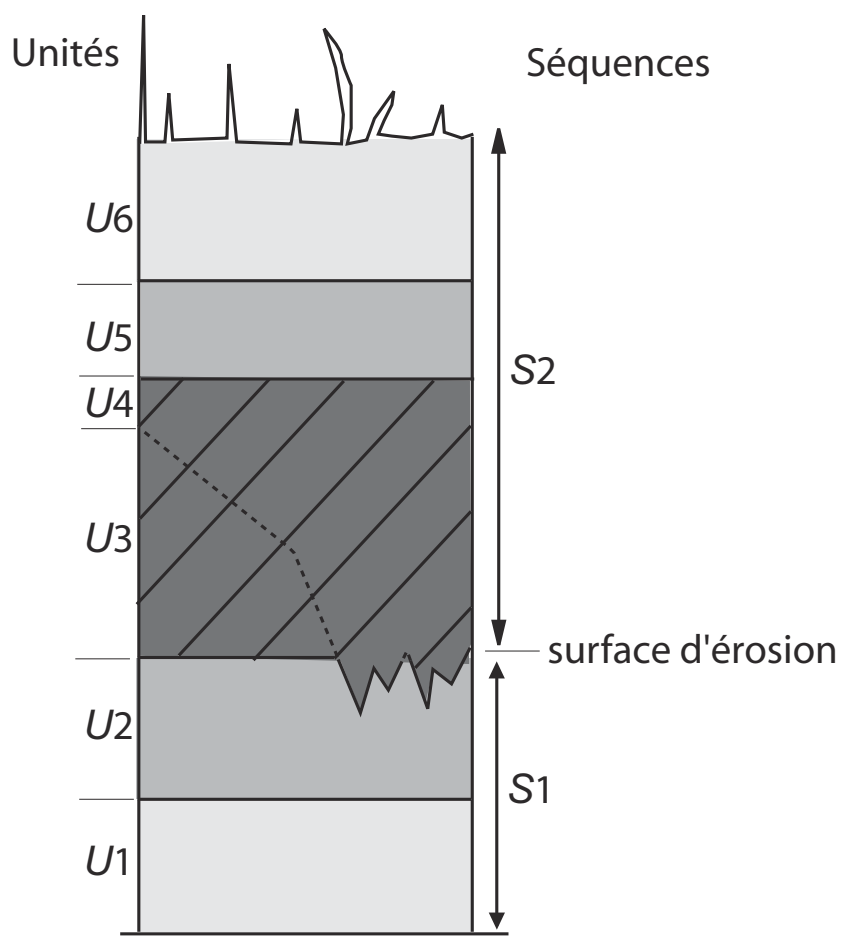

Figure 2 : Profil schématique représentant une succession d'unités pédologiques et sédimentaires $(U)$ regroupées en séquences génétiques $(S)$ qui correspondent à des phases dynamiques homogènes de sédimentation ou de pédogenèse.

Figure 2: Theoretical profile of a succession of pedo and sedimentary units $(U)$ which are related to genetic sequences $(S)$, each sequence corresponds to a homogeneous sedimentation or pedogenesis phase.

La taille et l'hétérogénéité des sites de Vitry-sur-Orne et Bliesbruck ont nécessité l'analyse de deux profils pédologiques de référence. Plusieurs types de paléosols ont été observés (tableaux 1 et 2). Des réducti-rédoxysols et fluvisols de fond de vallon et de plaine alluviale (Ay-sur-Moselle, Saint-Epvre, Le Borny, Lesmesnils, Bliesbruck) indiquent une forte oxydo-réduction. Des luvisols caractérisés par un horizon BT typique sont reconnus à Sarrebourg et en Forêt de Haye. Ils sont parfois tronqués comme à Imling. Un jeune rendisol de pente s'est développé sur des marnes calcaires à Solgne. Enfin, des sols plus ou moins brunifiés sont observés à Illange et Ennery. La séquence basale peut être remaniée soit en un colluviosol plus riche (Vitry-sur-Orne, Bouxière, Haut-Clocher) ou moins riche (Varrinchâtel) en artéfacts roulés, soit en un anthroposol totalement artificiel (chenal comblé à Moyenvic). Des colluviosols plus ou moins anthropisés recouvrent ces séquences basales (Illange, Vitrysur-Orne, Ay-sur-Moselle, Le Borny, Solgne, Saint-Epvre, Bouxière, Bliesbruck, Haut-Clocher, Imling).

À Illange et Bouxière, des fentes de dessiccation formant de vagues polygones de quelques décimètres de diamètre (fig. 3, a, b) présentent un remplissage limoneux blanchâtre appauvri en fer et argile. En lame mince, on y observe divers éléments organiques associés à des fragments remaniés d'horizon $\mathrm{BT}$ issus d'un luvisol érodé en amont. Ces fentes sont soulignées par une auréole d'oxydation liée à la migration $\mathrm{du}$ fer et du manganèse lors d'alternances de phases de forte sécheresse et d'humidité (Roger Langohr, com. pers.). À Bouxière, elles apparaissent à la surface de décapage du niveau gallo-romain et descendent jusqu'aux niveaux supérieurs du Bronze Final. De petites micro-fissurations sont également observées en lame mince dans les revêtements poussiéreux du colluviosol de Solgne. La formation de ces fentes pourrait avoir accompagné l'assèchement des fonds de vallées humides (Illange, Bouxière, Solgne) à la suite de l'abaissement de la nappe phréatique (Lesmesnils).

Sur l'ensemble des sites (tableaux 1 et 2), on observe la présence de colluvions accumulées dans les dépressions naturelles ou artificielles, dans les zones basses et à l'amont des micro-reliefs. Ceci indique une intense érosion des sols de pente. De nombreux indices dans ces colluvions évoquent une origine anthropique. Ainsi, les phytolithes associés aux graminées/herbacées et les revêtements argileux poussiéreux témoignent d'une mise à nu de la surface du sol due à la déforestation. Sur certains sites, des résidus organiques humifiés, des nodules phosphatés et des artéfacts anthropiques remaniés et roulés révèlent des apports de fumier (Vitry-sur-Orne, Ay-sur-Moselle, Saint-Epvre). Des déjections d'herbivores attestent la présence de bétail dans un champ (Borny) ou un chemin creux (Vitry-sur-Orne). Le travail du sol est avéré à Moyenvic (labour) et Bliesbruck (jardinage). Enfin, à Saint-Epvre, l'étude micromorphologique d'un billon a démontré son rôle majeur dans la mise hors d'eau du sol humide local, pour gagner des espaces agricoles (Gebhardt, 2008a). L'ensemble de ces données confirme une forte pression anthropique. 

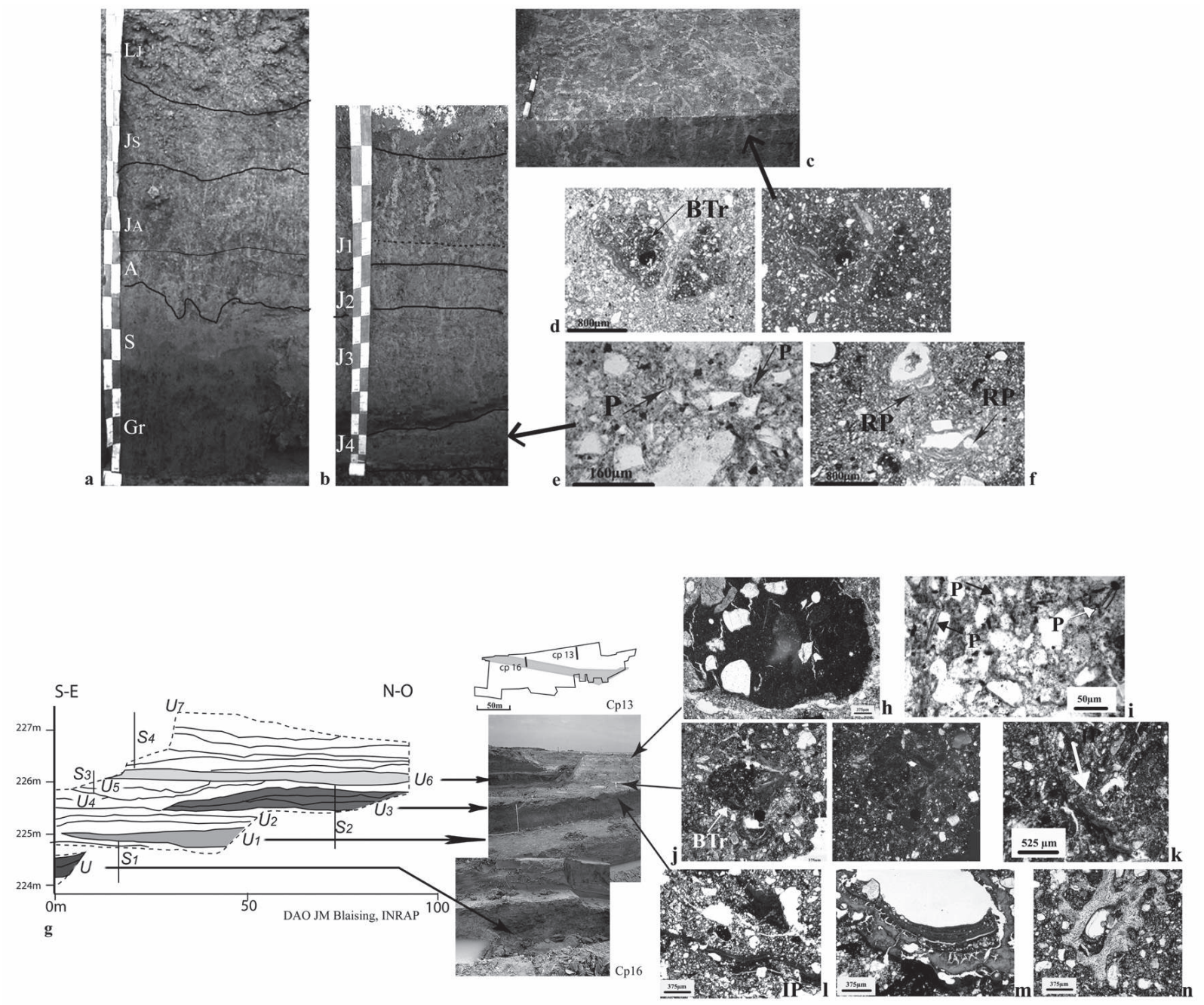

Figure 3 : (Voir planche couleur) Coupes étudiées et caractéristiques micromorphologiques; Illange, a. sondage SD5049/Zone4, séquence colluviale inversée $\left(J_{A} J_{S}\right)$ sur un brunisol $(A / S / G r), L_{J}$ : horizon de labour, $J_{S}$ : horizon colluvial supérieur, $J_{A}$ : horizon colluvial formé à partir de l'érosion de l'horizon $\mathrm{A}$ en amont, $\mathrm{A}$ : horizon organo-minéral, $\mathrm{S}$ : horizon structural, Gr : horizon gleyifié; Bouxière-sousFroidemont, b. colluviosol en limite de berge, $\mathrm{J}_{4}$ : horizon colluvionné, $\mathrm{J}_{3} \mathrm{~J}_{2} \mathrm{~J}_{1}$ : horizons colluvionnés avec apports alluviaux; c. réseau de fentes vues en plan; d. remplissage des fissures appauvri en fer avec un fragment remanié d'un horizon BT (BTr), Lumière Naturelle (LN), Lumière Polarisée (LP); e. abondants phytolites (P) (LN); f. revêtements argileux poussiéreux (RP) (LN); Saint-Epvre, g. coupe synthétique du remplissage du vallon, localisation des coupes 16 et 13 par rapport au paléochenal (en bleu, Blaising et al., 2011), S (1, 2, $3 \ldots)$ : séquences génétiques regroupant plusieurs unités pédologiques et sédimentaires $U(1,2 \ldots)$, en bleu foncé $(U)$ : horizon organique daté de 9520 +/-70 BP (9160-8700 cal BC), en orangé (U1) : 2780+/-30 BP (1000-850 cal BC) au Bronze final, en rouge (U3) : Haut Moyen Âge, en jaune (U6) : An Mil; h. fragment de céramique roulé (LN), i. phytolites (LN); j, fragment de sol remanié (LN/LP), k/l. intercalations poussiéreuses (LN), m. revêtements phosphatés (LN/LP), n. fragment osseux (LN).

Figure 3: (See colour plate) Profiles and soil micromorphological characteristics; Illange, a. SD5049/Zone4, reversed colluviated sequence (JSJ) on a preserved soil $(A / S / G r), L_{j}$ ploughed layer, $J_{S}$, upper colluviated horizon, $J_{A}$ : colluviated horizon from eroded A horizon, A: organo-mineral horizon, S: structural horizon, Gr: gley horizon; Bouxière-sous-Froidemont, $b$. colluviated soil profile on river bank, $J_{4}$ : colluviated horizon, $J_{3} \mid$ $J_{2} \bigvee_{1}$ : colluviated horizons with alluvial input; $c$. fissures observed from the surface; $d$. fissures with bleached infill and reworked fragments of a BT horizon (BTr) - Plain Polarized Light (PPL), Cross Polarized Light (XPL); e. numerous phytolithes (P) (PPL); f. clayey dusty coatings (RP) (PPL); Saint-Epvre, g. synthetic sequence of the valley infill, location of profiles 16 et 13 and palaeo-channel (in blue, Blaising et al., 2011), S (1, 2, 3...): genetic sequences composed of several pedo-and sedimentary units $U(1,2 \ldots)$, organic horizon in dark blue $(U)$ dated from -9520+/$70 \mathrm{BP}(9160-8700 \mathrm{cal} \mathrm{BC})$, in orange $\left(U_{1}\right): 2780+1-30 \mathrm{BP}(1000-850 \mathrm{cal} \mathrm{BC})$, in red $\left(U_{3}\right):$ Early Medieval, in yellow $\left(U_{6}\right):$ Year 1000 ; $h$. rolled sherd fragment (PPL/XPL), i. phytolithes (PPL), j. reworked soil fragment (PPL/XPL), k/l. dusty intercalations (PPL), m. phosphatic accumulation in pores (PPL/XPL), n. bone fragment (PPL). 


\section{ESSAI DE RECONSTITUTION DES GRANDES PHASES D'ÉROSION DES SOLS EN LORRAINE AU COURS DE L'HOLOCÈNE}

\section{Les premiers impacts anthropiques au Néolithique}

Si quelques-uns des sites archéologiques lorrains les plus anciens attestent d'une bonne stabilité du paysage au cours de l'Atlantique et du Subboréal (Illange, Ennery), d'autres révèlent des transferts sédimentaires qui viennent enrichir les sols de fond de vallée dès le Néolithique (Ay-sur-Moselle). Ils témoignent d'un impact anthropique, limité aux versants de secteurs par la suite fortement érodés (Vitry-sur-Orne). Des phases d'érosion précoce sont également signalées à Pagnysur-Moselle et Vandière (Carcaud, 1992), à Remerschen (Baes et Fechner, sous presse) et à Marsal (Fechner et al., sous presse) en Lorraine. Sur les loss d'Alsace (Ertlen et al., 2013), une quarantaine de centimètres d'horizons supérieurs de certains paléosols a pu colluvionner localement dès le Néolithique Final (Gebhardt, 2011d). Cela s'accorde avec les travaux palynologiques de Vergne (2004) qui montrent une détérioration du couvert végétal lorrain s'affirmant progressivement au cours du Subboréal. Une phase majeure d'anthropisation affecte des sites de basse altitude en Lorraine septentrionale, marquée par l'explosion des plantes rudérales autour de $4900 \mathrm{cal} \mathrm{BC}$ (Ruffaldi, 1999; AA-20328: $6065 \pm 65$ BP, com. pers.). Dans les Hautes Vosges, les premières céréales sont attestées vers 4800-4490 cal BC (5800 \pm 70 BP) à Moselotte et aux Trois Fours (Vergne, 2004).

L'influence anthropique, marquée dès le milieu du Néolithique par un détritisme alluvial ponctuel succédant à une sédimentation plus organique prévalant jusqu'à la fin de l'Atlantique, est également ressentie dans les régions limitrophes de la Lorraine telles que les vallées du bassin de Paris (Pastre et al., 2002), la région lyonnaise (Franc, 2005), la vallée du Rhône (Delhon et al., 2009) et l'Europe Centrale (Thiemeyer et al., 2005; Dotterweich, 2008).

\section{Une extension des défrichements pendant l'Âge du Bronze}

À l'Âge du Bronze, au cours de l'optimum climatique tardif du Subboréal (OAB, Optimum de l'Âge du Bronze; fig. 6), les berges sont occupées à Ennery (vallée de la Moselle), Bouxière (vallée de la Seille) et Saint-Epvre (vallée de la Nied) (fig. 1 et tableau 1). Des phases locales d'érosion marquées par des colluvions de versant étendues et épaisses sont décelées dès le début du Subatlantique. Ces colluvions s'accumulent au bas des pentes remplissant les thalwegs encore actifs peu de temps avant (Saint-Epvre). Ces apports limono-sableux indiquent le déboisement et la mise en culture des pentes. En effet, la recrudescence des apports limoneux vers 2600-2300 cal BC (4000 BP) coïncide avec une ouverture des paysages à la fin du Néolithique et au début de l'Âge du Bronze, confirmée par l'analyse pollinique (Vergne, 2004). Cette évolution est également ressentie dans la dynamique alluviale de la Moselle (Carcaud, 1992) et concorde avec l'apparition, dans le paysage naturel forestier de la fin du Subboréal, des premiers indices de céréaliculture en plaine (Koenig et Ruffaldi, 2007). Des traces de défrichement par le feu sont également révélées dès l'Âge du Bronze sur les sommets vosgiens (Goepp et al., 2008). En même temps, un pôle d'expansion humaine semble se développer à l'Âge du Bronze au sud de Nancy, mais l'occupation de l'espace lorrain ne paraît toutefois pas continue avant le Bronze final (Buzzi et al., 1993; Koenig et Ruffaldi, 2007).

Là encore, la Lorraine semble en accord avec les modèles des régions limitrophes (Kuzucuoglu et al., 1992; Braillard et al., 2002; Leopold et Völkel, 2006; Dotterweich, 2008; Doyen et al., 2013). Les premières mises en culture de l'Âge du Bronze final semblent ici affecter localement, mais sensiblement, les horizons supérieurs organiques fragiles des sols dès la fin du Subboréal.

\section{La crise sédimentaire de l'Âge du Fer}

Ce n'est qu'au cours de l'Âge du Fer que l'Homme semble véritablement impacter les paysages lorrains, même si l'anthropisation de l'environnement se fait sentir dès les périodes antérieures. D'importantes colluvions, corollaires d'une exploitation des versants, comblent les thalwegs et les dépressions, que ce soit sur le plateau lorrain, les vallées ou les pentes du massif des Vosges. Sur de nombreux sites, les sols sont tronqués (Imling, Varrinchâtel). À Bouxière, les occupations remontent plus haut sur les berges et les pentes, tandis que les fonds de vallée toujours humides et parcourus par des cours d'eau encore actifs, bien que localement pâturés (Le Borny), ne présentent pas d'installation humaine permanente. Ce schéma semble se reproduire en Alsace sur les bords de l'Ill (Nilles et al., 2010). L'érosion anthropique reste localisée mais précoce sur le versant lorrain des Vosges gréseuses. Les fragments roulés d'horizon illuviaux (BT) observés quasiment systématiquement au sein des sédiments colluvionnés attestent de l'importante épaisseur de sol affectée par l'érosion. Sur les pentes gréseuses fragiles de Varrinchâtel, l'épaisseur des horizons érodés entre les premières occupations du site $\mathrm{au} \mathrm{VI}^{\mathrm{e}} \mathrm{s}$. av. J.-C. et l'enfouissement du profil sous le rempart du Ive s. avant J.-C. (Gebhardt et al., 2009) permet d'estimer la vitesse d'érosion à $30 \mathrm{~cm}$ en un siècle. Ce taux est comparable à celui observé 

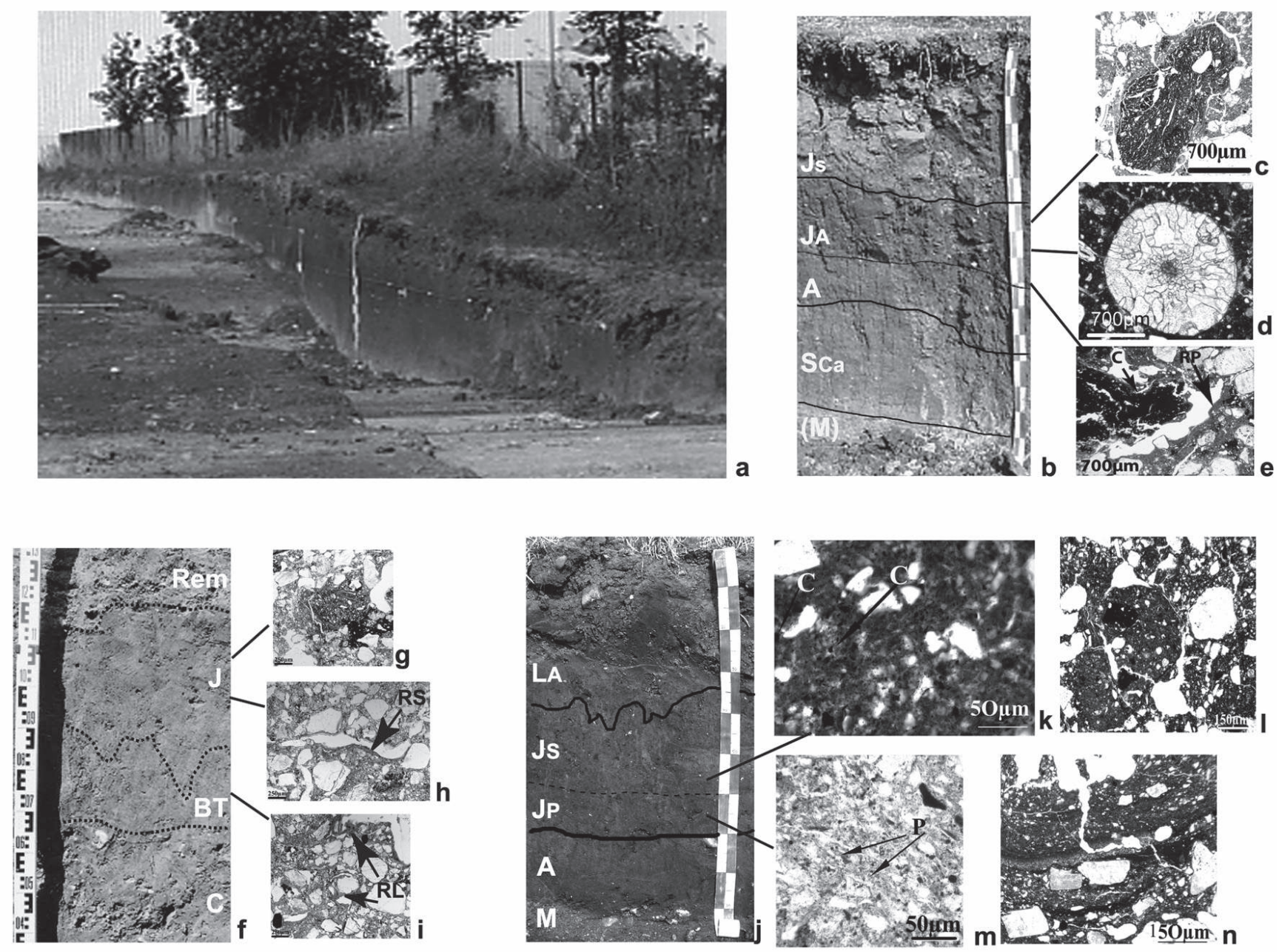

Figure 4 : (Voir planche couleur) Coupes étudiées et observations micromorphologiques; Ennery, a. vue générale du transect préservé dans un creux du relief; b. séquence colluviale inversée $\left(J_{A} J_{S}\right)$ sur un fluvisol brunifié $\left(A / S_{C 2} /(M)\right.$; c. fragment de céramique émoussé et roulé (LN); d. bionodule de calcite émoussé et roulé (LP); e. fragment de charbon (C) et revêtements argileux poussiéreux (RP; LN) ; Imling, f. luvisol tronqué (BT/C) par des rigoles d'érosion et recouvert d'un colluviosol (J), sous des remblais de démolition (Rem); g. fragment de sol allochtone (LN); h. revêtement argilo-silteux poussiéreux peu épais (RS; LN), i. revêtements argileux limpides (RL) de l'horizon illuvial (BT) en place (LN); Ay-sur-Moselle, j. fluvisol peu évolué, développé sur les formations alluviales sablo-limoneuses meubles de débordement $(\mathrm{A} / \mathrm{M})$ et recouvert par un anthroposol cumulique $(\mathrm{Jp})$ très sombre puis un colluviosol $(\mathrm{Js})$; $k$. fond matriciel organique avec de nombreux micro-fragments charbonneux $(\mathrm{C})$; 1. fragment de sol réticulé, roulé (LN); m. déjection d'herbivore avec nombreux phytolites (LN), n. fragment de litage relictuel (LN).

Figure 4: (See colour plate) Profiles and soil micromorphological characteristics; Ennery, a. general view of the transect in a depression: b. reversed colluvial sequence (JA\S) on the preserved soil $(A / S C a /(M))$; c. rounded and rolled ceramic fragment (PPL); $d$. rolled and rounded calcite bionodule (XPL); e. charcoal fragment (C) and dusty clay coating (RP; PPL); Imling, f. luvisol (BT/C) truncated by erosion gullies and covered by a colluviosol (J), under man-made debris (Rem); g. allochtonous soil fragment (PPL); h. clayey-silt dusty thin coating (RS; PPL); i. limpid clay coatings (RL) from the undisturbed BT horizon; Ay-sur-Moselle, j. fluvisoil developed on loamy-sandy overflow sediments (A/M) overlain by a dark anthroposoil (Jp) and a colluviosoil (Js); $k$. Ay-sur-Moselle, organic groundmass with numerous micro-charcoal (C) fragments (PPL); l. rolled reticulated soil fragment (PPL); $m$. numerous phytolithes (P) in an herbivorous dung (PPL), $n$. relictual bedding fragment (PPL).

sur les collines morainiques de l'est lyonnais $(30 \mathrm{~cm} /$ siècle; Franc, 2005) et en Bourgogne (15 cm par siècle, Petit et al., 2006). Sur certains sommets boisés des Vosges moyennes, l'ouverture du milieu forestier permet l'explosion de la lande à Callune (Guillet, 1972) et s'accompagne d'une mise à nu du sol précédant l'occupation gallo-romaine comme au Wasserwald (Gebhardt, 2007a). Dans le secteur de La Bresse (Vosges), on note des traces d'abattage d'arbres à la fin de l'Âge du Fer et une remontée des nappes phréatiques favorisant le développement de prairies humides (Kalis et al., 
2006). Sur le Rossberg (Alsace), la présence de charbons de genévrier commun révèle des prairies sous pâturées ou en voie d'abandon à La Tène Finale (Goepp et al., 2008). Au pays de Sarrebourg, l'analyse palynologique du remplissage de mardelles révèle le développement de prairies, sans doute favorisées par le pâturage de sols érodés devenus impropres à la culture à la fin de l'Âge du Fer (Etienne et al., 2011, 2013).

\section{Assèchement des fonds de vallée pendant la période gallo-romaine}

Le passage à l'Antiquité gallo-romaine semble se faire sans transition sur les sites étudiés. Comme en Allemagne (Leopold et Volkel, 2006) et en Franche-Comté (Sordoillet et al., 2013), les populations lorraines romanisées ont composé avec des sols appauvris et des paysages largement transformés par leurs prédécesseurs. À Imling, la mise à nu par l'érosion de l'horizon illuvial BT puis son recouvrement par des dépôts colluvionnés avant l'installation d'une villa au $\mathrm{I}^{\text {er }}$ s. apr. J.-C., témoigne de cet héritage (Gebhardt et al., 2009). Cette période coïncide avec l'amélioration climatique du Petit Optimum Romain (POR, fig. 6). Les déficits hydriques semblent assez importants pour provoquer dans les sols la formation de fentes de retrait caractéristiques (Illange, Bouxière), voire la fissuration des revêtements argileux (Solgne) et un assèchement rapide, au moins saisonnier, permettant l'exploitation de terres alluviales jusque-là très humides (Bliesbruck, Lesmesnils). Dans le bassin de SaintDié, le paysage boisé de la vallée de la Fave subit les premiers assauts de l'Homme au profit du noyer et des cultures de céréales et de chanvre (Allenet, 2008). Le cours d'eau se recentre dans son lit mineur, n'excluant pas des débordements limoneux fins saisonniers plus loin sur les berges et la formation de tourbes dans les chenaux résiduels.

Malgré des crues encore récurrentes attestées dans d'autres régions (Salvador et al., 2002), la conquête des plaines alluviales lorraines est peut-être aussi, à l'instar des vallées du Rhône, de la Saône ou du Doubs, initiée par le besoin d'espaces et le développement d'une agriculture productiviste (Berger et Bravard, 2012). En effet, des travaux de drainage (Saint-Epvre) ou d'empierrement (Haut-Clocher) permettent d'accélérer la mise en valeur des derniers terrains encore humides. À Moyenvic, le labour est attesté dans un chenal comblé (Gebhardt, 2004a, 2010b). En Forêt de Haye, la mise en place de terrasses permet localement de contenir l'érosion.

\section{Relative stabilisation médiévale des paysages}

En parallèle à la poursuite d'une emprise forte et continue sur le milieu, les espaces agraires se structurent au cours du Moyen Âge (Vallange). Les labours en billons (Saint-Epvre) atténuent l'humidité de certains fonds de vallons comblés, comme aux abords de la Fave où se développent des prairies bombées encore visibles aujourd'hui (Gebhardt, 2006). Dans les Vosges, on note une oscillation entre des phases agropastorales et de retour de la forêt, tandis que la remontée du niveau des nappes phréatiques favorise l'extension des tourbières à La Bresse (Kalis et al., 2006). Sur les pentes limoneuses lorraines, transferts sédimentaires et surfaces érodées augmentent malgré un taux d'érosion stable $(15 \mathrm{~cm}$ en un siècle à Saint-Epvre). Une gestion rationalisée des espaces agraires est attestée par l'installation de parcellaires et la fixation des paysages par des haies, des prés et des friches; elle semble accompagner la pression humaine grandissante sur les sols. Certains massifs forestiers sont affectés par des pressions socio-économiques telles les exploitations salines dans le massif du Römersberg par exemple (Etienne et al., 2010). Caractérisée à la fois par des fluctuations climatiques notables au cours du millénaire médiéval (fig. 6) et des aléas historiques majeurs (conflits, épidémies), la dynamique paysagère médiévale reste difficilement généralisable sans une confrontation avec les recherches historiques de ces secteurs (Garnier, 2004).

\section{Discussion}

Ce premier bilan de l'évolution pédo-sédimentaire en Lorraine durant l'Holocène se heurte à plusieurs limites : - La représentativité et le maillage des sites

Les sites étudiés, qui présentent a priori un intérêt archéologique, offrent l'avantage de permettre de dater les phases de colluvionnement. Toutefois, cet échantillonnage pédosédimentaire ne résulte pas d'un inventaire programmé de tous les types de contextes archéologiques ou géomorphologiques lorrains. Au contraire, les rares sites analysés par l'archéologie préventive ont été découverts au hasard des aménagements du territoire actuels localisés surtout dans le sillon lorrain. Le maillage de ces sites ne couvre pas l'ensemble des milieux et ne garantit pas la représentation continue des processus dans le temps. Malgré ces limites, cette étude montre le potentiel lorrain et ouvre la voie à des recherches ciblées. 

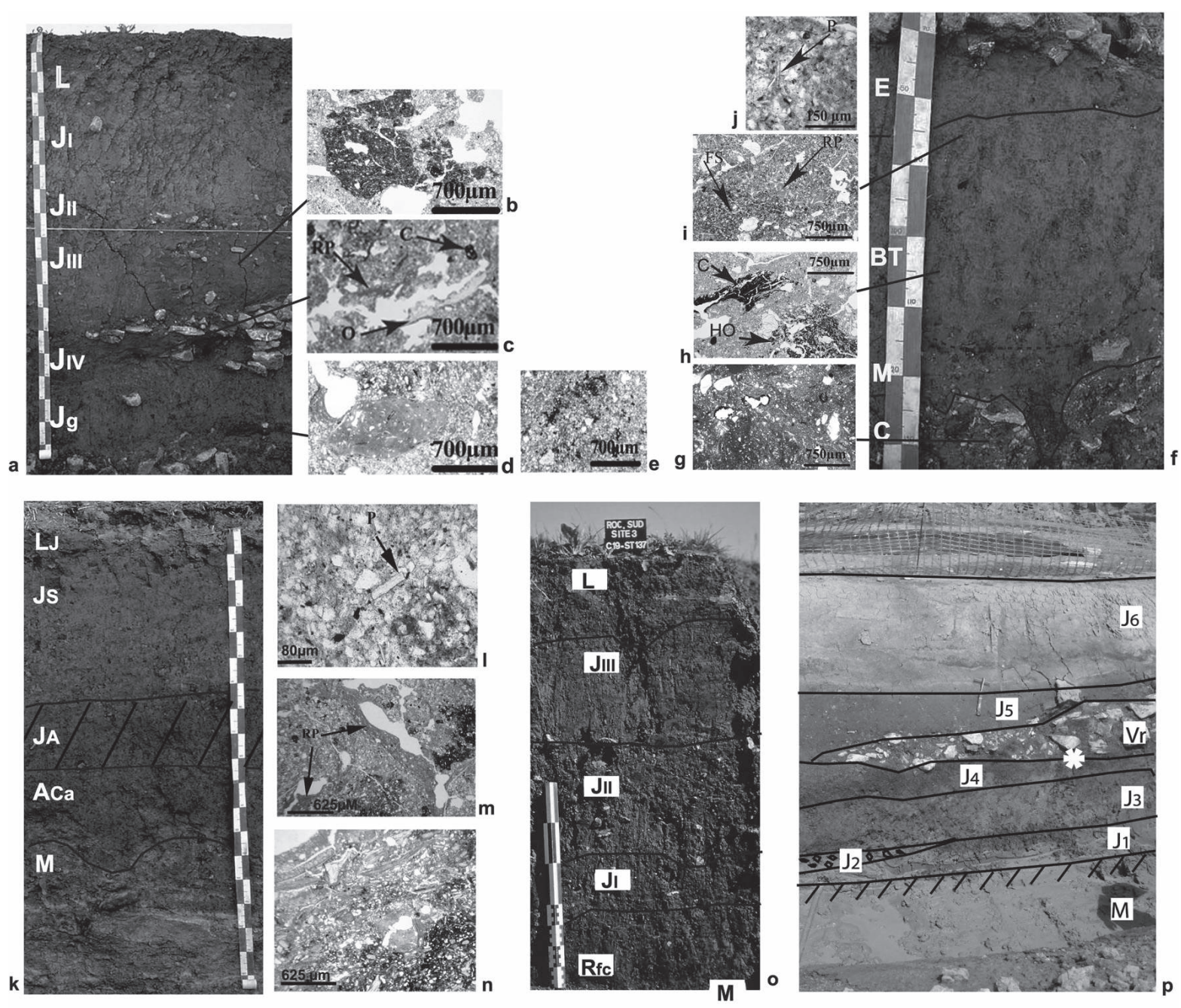

Figure 5 : (Voir planche couleur) Coupes étudiées et observations micromorphologiques; Le Borny, a. rédoxysol cumulique (Jg) sur marne ruisselée, recouvert par des remblais de carrière antique $\left(\mathrm{J}_{\mathrm{IV}}\right)$, puis par une séquence colluviale historique non datée $\left(\mathrm{L} / \mathrm{J}_{\text {III }}\left|\mathrm{J}_{\text {II }}\right| \mathrm{L}_{\mathrm{I}}\right)$; b. fragment roulé de poterie (LN); c. revêtement argileux poussiéreux (RP), charbons (C), os (O) dans remblais de carrière (LN), d. déjection d'herbivore (LN), e. imprégnation de fer dans la masse (LN); Sarrebourg, f. luvisol (E/BT/M/C) développé sur des plaquages de limon recouvrant une terrasse alluviale ancienne de la Sarre, g. aspect général du calcaire marneux altéré du substrat (LN), h. fragment d’horizon organique (HO) et charbon (C) (LN), i. fragment de sol organique remanié (FS) et revêtements argileux poussiéreux (RP) (LN), j. phytolite $(\mathrm{P})(\mathrm{LN})$; Solgne, $k$. rendisol développé sur un substrat marneux altéré et ruisselé $\left(\mathrm{A}_{\mathrm{C}_{2}} / \mathrm{M}\right)$, recouvert par une séquence colluviale $\left(\mathrm{J}_{\mathrm{A}} \backslash \mathrm{L} / \mathrm{J}_{\mathrm{S}}\right)$, l. phytolites $(\mathrm{P})(\mathrm{LN})$, m. revêtement argileux poussiéreux $(\mathrm{LN})$, n. argiles calcaires ruisselées (LN); Peltre, o. remplissage de fond de cabane en fosse creusé jusqu’à la dalle calcaire $(\mathrm{Rfc} / \mathrm{M})$, recouvert par deux séquences colluviales $\left(\mathrm{J}_{\mathrm{I}} \mathrm{J}_{\mathrm{II}} \mathrm{puis} \mathrm{L} / \mathrm{J}_{\mathrm{III}}\right)$; Haut-Clocher, p. succession stratigraphique du vallon avec de bas en haut : un rédoxysol $(\mathrm{M})$ sous des apports gravelo-sableux tardiglaciaires $\left(J_{1} \mathrm{~J}_{2}\right)$, des limons colluvionnés protohistoriques $\left(\mathrm{J}_{3} \mathrm{~J}_{4}\right)$, une voie antique $(\mathrm{Vr})$ et des colluvions post-romaines $\left(\mathrm{J}_{5}\left(\mathrm{~J}_{6}\right)\right.$; l'étoile localise le charbon daté prélevé au sommet des limons $\left(\mathrm{J}_{4}\right)$.

Figure 5: (See colour plate) Profiles and soil micromorphological characteristics; Le Borny: a. cumulic redoxysol (Jg) buried under Roman dump $\left(J_{I V}\right)$ and historical undated colluvium $\left(L / L / J_{I I} / J_{I I I}\right)$; b. sherd fragment (PPL); c. layered dusty clay coating (PPL); d. herbivorous dung (PPL); e. iron incrustation in the groundmass (PPL); Sarrebourg, f. luvisol (E/BT/M/C) developed in a patchy loamy unit over the alluvial deposits of an old terrace of the Sarre River, g. general view of the weathered marle at the top of the bedrock (PPL), h. charcoal (C) and organic horizon fragment (HO) (PPL), i. reworked organic soil fragment (FS) and dusty clay coating (RP) (PPL), j. phytolithe (P) (PPL); Solgne: k. rendisoil developed in the washed marly bedrock $\left(A_{C a} / M\right)$ and overlain by a colluvial sequence $\left(J_{A} \backslash L / J_{S}\right)$, l. phytolithes $(P)(P P L), m$. dusty $(R P)$ clayey coatings (PPL), n. washed calcareous clay (PPL); Peltre, o. infeeling of a grubenhaus dug in the calcareous bedrock (RfclM), overlain by two colluvial sequences $\left(J_{I} \bigcup_{I I}\right.$ then $\left.L / J_{I I I}\right)$; Haut-Clocher, p. stratigraphic succession of the valley, from the bottom up: redoxy soil (M) overlain by a late-glacial sandy gravel $\left(J_{1} \backslash J_{2}\right)$, protohistorical colluviated loam $\left(J_{3} \bigvee_{4}\right)$, a Roman road (Vr) and historic post-Roman colluvium $\left(J_{5} \bigvee_{6}\right)$; star: location of the dated charcoal, at the top of the loam $\left(J_{4}\right)$. 


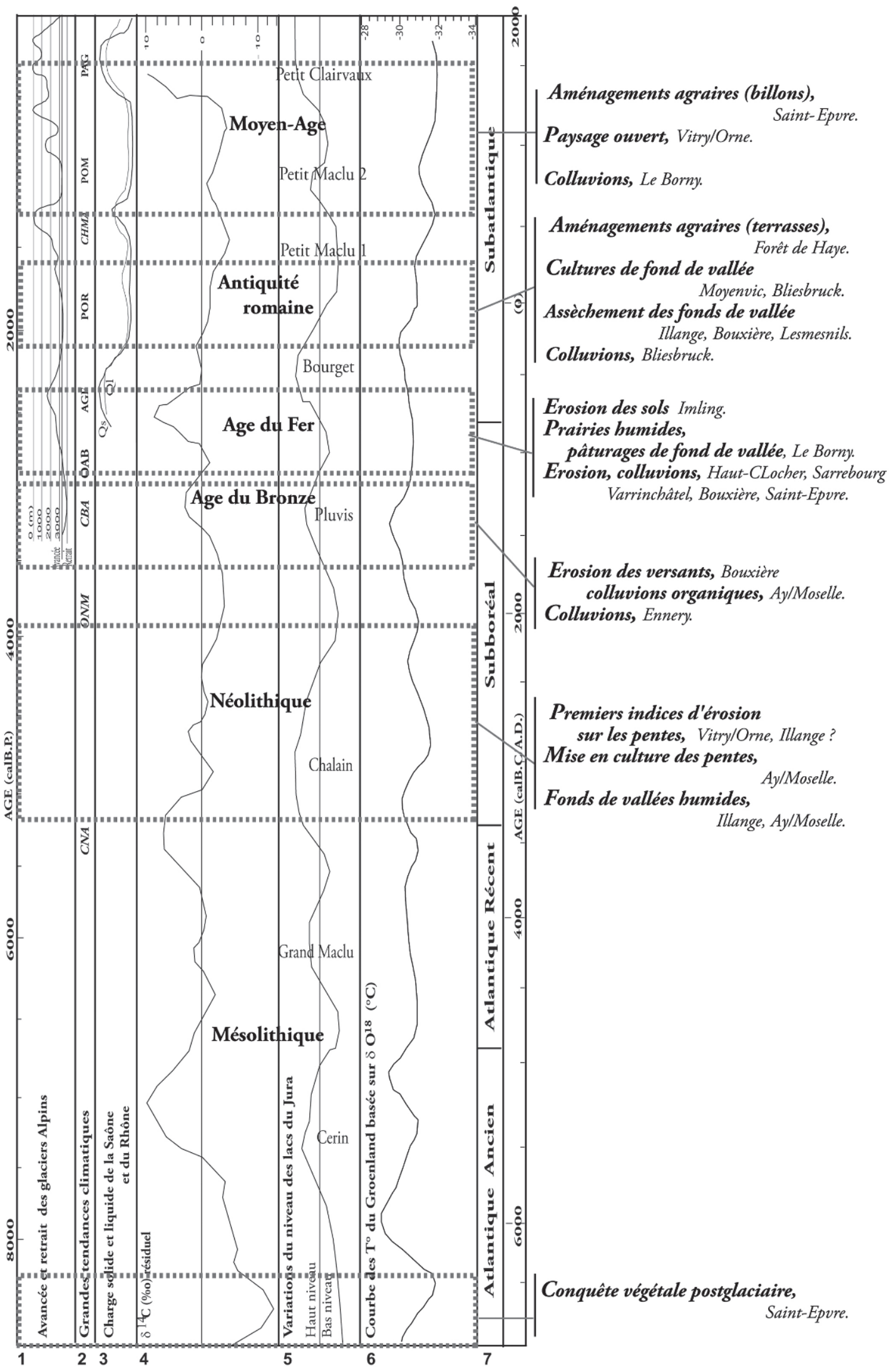

ArcheoSciences, revue d'archéométrie, 38, 2014, p. 7-29 
Figure 6 : (Page précédente) Corrélation des séquences pédo-sédimentaires des sites de Lorraine (8) avec les phases paléo-climatiques et archéologiques de l'Holocène définies par différents marqueurs : 1. fluctuations des glaciers alpins (Holzhauser et al., 2005); 2. fluctuations climatiques reconnues à l'échelle de l'Europe (Magny, 1995) (CNA : Crise du Néolithique Ancien, AGF : Age Glaciaire du Fer, PAG : Petit Age Glaciaire, et OAB : Optimum de l'Age du Bronze, POR : Petit Optimum Romain, POM : Petit Optimum Médiéval). D'autres fluctuations secondaires peuvent s'y intercaler (CNA : Crise du Néolithique Ancien, ONM : Optimum du Néolithique Moyen, $C B A$ : crise du Bronze Ancien, $C H M A$ : crise du Haut Moyen Âge) ; 3. charge solide et liquide de la Saône et du Rhône (Bravard, 1997); 4. carbone résiduel (van Geel et Magny, 2002) ; 5. variations du niveau des lacs du Jura (van Geel et Magny, 2002); 6. courbe vectorielle, à moyenne glissante sur 500 ans, des températures $\left({ }^{\circ} \mathrm{C}\right)$, établie à partir de $\delta^{18} \mathrm{O}$ de la carotte de glace GISP 2 (Groenland, d'après Wanner et al., 2011); 7. chronozones palynologiques (Mangerud et Berglund, 1978).

Figure 6: (Previous page) Correlation of the pedo-sedimentary sequences observed at the sites of Lorraine (8) with multi proxy paleoclimatic and archeological phases of Holocene: 1. Alpine-glaciers fluctuations (Holzhauser et al., 2005); 2. main european climatic phases (Magny, 1995): CNA: Ancient Neolithic crisis, AGF: Iron Age Crisis, PAG: Small Ice Age, and OAB: Bronze Age Optimum, POR: Small Romain Optimum, POM: Small Medieval Optimum. Other secondary fluctuations can be intercalated: CAN: Ancient Neolithic Crisis, ONM: Middle Neolithic Optimum, CBA: Ancient Bronze Crisis, CHMA: Early Middle Age Crisis; 3. liquid and solid charge of the Saône and Rhône rivers (Bravard, 1997); 4. residual carbon variations (van Geel et Magny, 2002); 5. Jura lakes level (van Geel et Magny, 2002); 6. temperature reconstruction $\left({ }^{\circ} \mathrm{C}\right)$ based on $\delta^{18} \mathrm{O}$ data from the GISP 2 ice core (Groenland, spline: $500 \mathrm{yr}$ cutoff period, after Wanner et al., 2011); 7. palynological chronozones (Mangerud et Berglund, 1978).

\section{- L’imprécision des données chronologiques disponibles en Lorraine}

Sauf dans le cas de rares implantations humaines in situ, la datation des processus évoqués repose sur des témoins archéologiques remaniés qui rendent, comme le souligne Leveau (2007), les calages chronologiques des séquences sédimentaires imprécis. Dans le cas de colluvions recouvrant un paléosol, le contenu archéologique donne soit un âge minimal au processus d'érosion à l'origine des colluvions, soit un âge maximal, en cas d'inversion stratigraphique. De plus, les grandes phases d'implantation humaine s'étalant sur plusieurs siècles, voire plusieurs millénaires, les témoins observés ne représentent en fait qu'une durée limitée, de l'ordre de quelques générations, au sein des phases archéologiques. L'âge et la durée des phases d'érosion ou de sédimentation reconstituées se situent donc, en l'absence de datation, dans un laps de temps non connu au cours de ces phases d'implantation humaine.

- Essai d'intégration des premiers acquis pédo-sédimentaires lorrains aux échelles climatiques de référence

La temporalité environnementale demande à être harmonisée (Bertrand, 2002). Les travaux les plus récents, en Europe occidentale, basés sur l'analyse d'enregistrements en domaines lacustre, glaciel et fluviatile (Bravard, 1997; Van Geel et Magny, 2002; Holzhauser et al., 2005), permettent aujourd'hui de définir pour l'Holocène six phases climatiques principales. Elles se structurent selon une alternance de périodes plus fraîches et humides assorties d'un hydro-dynamisme élevé et d'une remontée des nappes phréatiques intercalés avec des périodes plus chaudes et sèches. Ces fluctuations sont également bien reconnues dans les enregistrements glaciaires et marins à l'échelle globale (Davis et al., 2003; Mayewsky et al., 2004; Wanner et al., 2011 et le site HOCLAT de Wanner et Ritz). Toutes ces données nouvelles complètent et nuancent le premier modèle, toujours en vigueur, basé sur le croisement d'études biostratigraphiques et radiochronologiques à l'origine du découpage de l'Holocène en cinq chronozones (Mangerud et al., 1974; Mangerud et Berglund, 1978) allant du Préboréal au Subatlantique (Blytt [1876] et Sernander [1908]). Seules les oscillations récentes concernent cet article (fig. 6).

En Lorraine, malgré des inconnues qui relèvent du flottement de certains calages chronologiques bornant les différentes phases archéologiques, la confrontation des données pédo-sédimentaires présentées ici avec les référentiels climato-sédimentaires les plus récents montrent cependant certaines concordances. Ainsi, la détérioration climatique signalée dans les enregistrements d'Europe occidentale vers l'an 1000 avant J.-C. (avancée des glaciers alpins, augmentation de la charge solide du Rhône et de la Saône, remontée du niveau des lacs jurassiens) coïncide en Lorraine avec une crise sédimentaire enregistrée à l'Âge du Fer (phase AGF, fig. 6). Il en va de même pour l'optimum climatique (retrait des glaciers alpins, baisse de la charge solide du Rhône et de la Saône et du niveau des lacs du Jura) reconnu autour du changement d'ère (jusque vers le III $^{\mathrm{e}}$ siècle après J.-C. marqué par un retour de dégradation) et qui en Lorraine semble correspondre à une période de stabilisation des milieux (phase POR, fig. 6). La convergence entre les données pédo-sédimentaires lorraines et les enregistrements plus globaux pour les périodes plus anciennes ou plus récentes est pour le moment peu probante, ce qui limite la possibilité de calage chronologique des processus pédo-sédimentaires correspondants. Le Moyen Âge, qui a subi plusieurs fluctuations climatiques, reste, en Lorraine, encore trop peu 
concerné par les études pédo-sédimentaires pour permettre des corrélations pédo-climatiques.

\section{Crise climatique ou anthropique?}

La question posée ici concerne la part des processus dus aux facteurs naturels par rapport à celle induite par des processus liés aux activités humaines.

L'interaction entre homme et environnement est très étudiée par les chercheurs travaillant sur le pourtour méditerranéen (Bertoncello et al., 2011), secteur plus sensible aux pressions hydriques et aux interventions des sociétés humaines implantées depuis plus longtemps que dans les pays du nord de l'Europe. Dans le nord-est de la France, la question de ces interactions a déjà été abordée sur la base d'analyses polliniques (Gauthier et al., 2000; Richard et Ruffaldi 2004; Ruffaldi et al., 2006). Après la révélation de traces de céréaliculture proto-néolithique précoce très localisée, ces premiers travaux ne font état d'une ouverture franche des paysages de plaine qu'à la fin de l'Âge du Bronze. Au cours de l'Âge du Fer et de l'Antiquité, le paysage végétal ne s'anthropise alors qu'en altitude et les variations du couvert forestier sont plutôt mises sur le compte de soubresauts climatiques. Au Moyen Âge, des indices d'érosion agraire apparaissent dans le remplissage organique des mardelles. S'il semble prématuré à l'échelle de la seconde moitié de l'Holocène et dans l'état actuel encore trop morcelé des connaissances géo-archéologiques et environnementales de la région, d'étendre ce lien de causalité à la Lorraine; il est toutefois déjà possible de constater une interaction entre ces oscillations climatiques et les populations riveraines des cours d'eau. Ainsi, pour les sociétés lorraines à faible densité humaine antérieures à l'Âge du Fer, que l'on peut imaginer en symbiose avec l'environnement et réparties selon des mosaïques paysagères aux ressources multiples selon le modèle normand (Lespez, 2012), l'influence de ces crises est certainement moindre. La maîtrise gauloise de l'extraction et du travail du fer, dont le minerai est déjà exploité en Lorraine (Leroy et al., 2000), a ensuite facilité la diffusion de nouveaux outils (araire, charrue, faux) qui ont permis, par de nouvelles pratiques agricoles, d'augmenter rapidement les surfaces exploitables et la productivité (Malrain, 2000). En Lorraine, comme ailleurs (Franc, 2005; Petit et al., 2006; Leopold et Völkel, 2006; Dotterweich, 2008; Goepp et al., 2008; Nilles et al., 2010) le défrichement progressif des derniers obstacles aux écoulements superficiels signe le décloisonnement des paysages ouverts dès la fin du Néolithique et donne le départ de la généralisation de l'érosion des sols de pente. C'est également sans doute cette pression humaine de plus en plus forte sur les sols qui explique le possible décalage entre les crises climatiques générales et certaines réponses érosives locales.

À l'Âge du Fer, l'impact agricole semble ainsi s'allier à l'une des plus puissantes et soudaines ruptures climatique de la seconde moitié de l'Holocène (Van Geel, 2012; Magny, 2012) pour déclencher l'instabilité des pentes. Les GalloRomains lorrains sont ensuite les premiers à investir des fonds de vallée de plus en plus secs, la maîtrise hydrologique des cours d'eau n'arrivant qu'au Moyen Âge.

Comme en Europe Centrale (Bastian et Bernhardt, 1993; Dotterweich, 2008), le rythme d'érosion des sols lorrains, dépendant à la fois des conditions géomorphologiques locales et des variations climatiques globales, reste sans doute également largement influencé par une dynamique des populations reflétant les aléas démographiques locaux et la technologie disponible. La tentative de réflexion diachronique sur l'évolution des terroirs au cours des périodes historiques (Blaising, 2002) et ce présent article apportent, pour les périodes plus anciennes, les premiers éléments de reconstitution de l'histoire des relations entre les variations environnementales et les mutations sociales au cours de la seconde moitié de l'Holocène de la Lorraine.

\section{CONCLUSION}

En Lorraine, une quinzaine de sites archéologiques a fait l'objet d'une analyse pédo-sédimentaire diachronique, qui s'appuie sur l'identification des artéfacts archéologiques et quelques datations au ${ }^{14} \mathrm{C}$. Les colluvions, qui recouvrent ou tronquent des sols enfouis, révèlent l'intervention, pendant la deuxième moitié de l'Holocène, de processus d'érosion que nous attribuons principalement aux activités de défrichement et d'exploitation agricole. L'érosion des sols de Lorraine semble débuter très localement dès l'Âge du Bronze, voire au Néolithique. Elle se généralise et s'accentue au cours de l'Âge du Fer où, combinée à une détérioration climatique, elle atteint les taux les plus forts. Si l'époque gallo-romaine correspond à une stabilisation relative du milieu et à l'occupation des fonds de vallée, une reprise de l'érosion semble caractériser le Haut Moyen Âge.

Cette première tentative de reconstitution régionale met en évidence les mêmes tendances érosives que dans les régions voisines (Alsace, Allemagne rhénane, Luxembourg, vallée du Rhône) où la phase fortement détritique de l'Âge du Fer coïncide avec une détérioration climatique et une accentuation du défrichement. Cette étude permet également de replacer l'évolution de ces quelques sites lorrains dans une réflexion globale de causalité. Les liens entre la variabilité environnementale, en particulier climatique, et 
la dynamique des peuplements et des mutations sociales au cours de la seconde moitié de l'Holocène restent néanmoins à développer en Lorraine, en systématisant les recherches pluridisciplinaires programmées sur le territoire et en engageant des synthèses archéologiques.

\section{Remerciements}

Ce travail n'aurait pas pu être mené sans les contributions et les diverses opérations d'archéologie préventive, programmées ou universitaires, effectuées en Lorraine sous la responsabilité de G. Allenet (palynologie, Vallée de la Fave), X. Antoine (Illange), J.-M. Blaising (Saint-Epvre, Peltre), K. Boulanger (Bouxière), S. Deffressigne (Bouxière), M. Dohr (Remomeix), M. Gazenbeck (Borny), F. Gérard (Vitry-sur-Orne), R. Judes (Haut-Clocher, Remomeix), J.-D. Lafitte (Moyenvic), J. Lebéchennec (Bliesbruck), M. Leroy (Forêt de Haye), A. Masquillier (Solgne), N. Meyer (Sarrebourg), L. Olivier (Marsal), M. Prévost (Solgne), P. Rohmer (Imling), F. Schembri (Lesmesnils), F. Thieriot (Ennery), L. Thomashausen (Ay-sur-Moselle, Illange), B. Triboulot (Varrinchâtel). Qu'ils soient ici tous chaleureusement remerciés. Les auteurs remercient également les deux lecteurs anonymes dont l'analyse minutieuse a permis d'améliorer le manuscrit initial.

\section{Bibliographie}

Allenet G., 2008. Étude palynologique. In M. Dohr, Remomeix, "Les Mennes Hières » Phase2. Rapport de Diagnostic, INRAP GE-Nord, SRA Lorraine.

Baes R., Fechner K., sous presse. A field or garden in the Early Neolithic village of Remerschen : pedological data and comparison with other sites in the Moselle area, in K. Fechner, Y. Devos, M. Leopold, J. Völkel (eds). Archaeology, soil- and life-sciences applied to houses, gardens and courtyards. Second volume of the proceedings of the session 'From microprobe to spatial analysis - Enclosed and buried surfaces as key sources in Archaeology and Pedology', European Association of Archaeologists, 12th Annual Meeting, Krakow-Poland, 19th to 24th September 2006. British Archaeological Records, International Series.

BaIze D., 1992. Le référentiel pédologique des principaux sols d'Europe. Technique et pratiques, INRA éditions.

Baize D., Jabiol B., 1995. Guide pour la description des sols. Techniques et pratiques, INRA éditions.
Bastian O., Bernhardt A., 1993. Anthropogenic landscape changes in Central Europe and the role of bio-indication. Landscape Ecology, 8, (2) : 139-151.

Becze-Deak J., Fechner K., Langohr R., 1995. Fouilles archéologiques d'Ay-sur-Moselle en 1992. Résultats des Analyses pédologiques faisant suite au rapport pédologique de terrain. Service Régional de l'Archéologie Lorraine.

Berger J.-F., Bravard J.-P., 2012. Le développement économique romain face à la crise environnementale : le cas de la Gaule narbonnaise. In J.-F. Berger (dir.). Des climats et des hommes. La Découverte, 269-290.

Bertoncello F., Berger J.-F., Carozza L., Braemer F., Depaepe P., 2011. Variabilité environnementale, mutations sociales : nature, intensité, échelles et temporalités des changements. Résumé des XXXII ${ }^{\mathrm{e}}$ rencontres Internationales d'Archéologie et d'Histoire d'Antibes, 20-22 octobre 2011.

Bertrand G., 2002. La discordance des temps. In H. Richard et A. Vignot (éd.). Équilibres et ruptures dans les écosystèmes depuis 2000 ans en Europe de l'Ouest. Actes du Colloque de Besançon, septembre 2000, 15-23.

Blaising J.-M., 2002. Anthropisation du milieu rural durant les périodes historiques en Lorraine. PCR DRAC Lorraine, SRA, INRAP GEN.

Blaising J.-M., Baccega S., Braguier S., Gazenbeck M., Gebhardt A., Leroyer C., Tegel W., Wiethold J., 2011. Saint-Epvre "Le Château" (57), Habitat médiéval (vII ${ }^{\mathrm{e}}$-XII ${ }^{\mathrm{e}}$ s. et Billons $\mathrm{XII}^{\mathrm{e}}-\mathrm{X}^{\mathrm{e}} \mathrm{s}$.), TGV Est, lot 34. Rapport Final d'Opération INRAP-GEN.

BLYTt A., 1876. Forsøg til en Theorie om Indvandringen af Norges Flora under vexlende regnfulde og tørre Tider. $N m f N$, bd. 21 .

BoËs E., Gebhardt A., 2007. Paléo-environnement et anthropisation des paysages en Alsace : une dynamique renouvelée? Bilan Scientifique Hors-Série, 1/2, SRA DRAC Alsace MCC, 167-170.

Bonneau M., Faivre P., Gury M., Hétier J.-M., Le Tacon F., 1978. Carte pédologique au 1/100 000 de Saint-Dié. Carte et notice explicative.

Bork H.R., LaNG A., 2003. Quantification of past soil erosion and land use / land cover changes in Germany, Long term hillslope and fluvial system modelling : Concepts and case studies from the Rhine river catchment. In A. Lang, K. Heinrich-Dikau (eds.). Lecture Notes on Earth Sciences, 101, Springer-Verlag, 231-239.

Braillard L., Guélat M., Pousaz N., Rachoud-Schneider A.-M, 2002. Interactions entre occupation humaine et milieux naturels dans le bassin de Délémont (Jura, Suisse) : l'Âge du Bronze, un point de rupture manifeste dans la dynamique sédimentaire et l'évolution de la végétation. In $\mathrm{H}$. Richard, A. Vignot (dir.), Équilibres et ruptures dans les écosystèmes durant les 20 derniers millénaires en Europe de l'Ouest. Annales littéraires, 
730. Série "Environnement et sociétés”, 3, PUF-C, Besançon, 213-222.

Bravard J.-P., 1997. Géo-archéologie des vallées alluviales de Rhône-Alpes depuis le Tardiglaciaire. In J.-P. Bravard, M. Prestreau (dir.). Dynamique du Paysage, entretiens de géoarchéologie, Lyon, novembre 1995. DARA, 129-150.

BRÜск D., KubINok J., 1999. Holozäne Landschaftsentwicklung des Blies bei Reinheim/Bliesbrück, Bulletin de la Société Préhistorique Luxembourgeoise, 20-21 : 301-312.

Bullock P., Fedoroff N., Jongerius A., Stoops G., Tursina T., BABEL U., 1985. Handbook for soil thin section description, Waine Research Publication.

Buzzi P., Carcaud N., Koenig M.-P., Weisrock A., 1993. Morphodynamique fluviale holocène et établissements humains protohistoriques en fond de vallée de la Moselle à Crévechamps (Lorraine Méridionale). Revue Géographique de l'Est, t. XXXIII, (4) : 281-295.

Carcaud N, 1992. Remplissage des fonds de vallée de la Moselle et de la Meurthe en Lorraine sédimentaire. Thèse de géographie, Université de Nancy II, France.

Cordier S., Harmand D., Losson B., Beiner M., 2004. Alluviation of the Meurthe and Moselle valleys (Eastern Paris Basin, France), lithological contribution to the study of the Moselle capture and Pleistocene climatic fluctuations. Quaternaire, 15, (1-2) : 65-76.

Courty M.A., Goldberg P., Macphail R.I., 1989. Soils and micromorphology in archaeology, Cambridge manuals in archaeology.

Davis B.A.S., Brewer S., Stevenson A.C., Guiot J., Data Contributors, 2003. The temperature of Europe during the Holocene reconstructed from pollen data. Quaternary Science Reviews, 22 : 1701-1716.

Decaulne A., 2007. Les éboulis, mise en place, dynamique et signification climatique : exemple de la gravière du Valtin. In S. Cordier, D. Harmand, S. Occhietti (dir.). Livret guide de l'excursion de l'AFEQ des 7-9 juin 2007, Lorraine, Luxembourg, Rhénanie-Palatinat, Sarre, 231-233.

Delhon C., Thiébault S., Berger J.-F., 2009. Environment and management of space during middle Neolithic in Southern France : evidences of agro-sylvo-pastoral systems in the middle Rhone valley. Quaternary International, 200 : 50-65.

Deschodt L., Salvador P.-G., 2006. Diversité des enregistrements tardiglaciaires et holocènes dans la vallée de la Deûle (59, Nord). In Ph. Allée and L. Lespez, (dir.). L'Érosion entre société, climat et paléoenvironnement. Table ronde en l'honneur du Professeur René Neboit-Guilhot, Clermont-Ferrant, P.U. Blaise-Pascal, collection "Nature et Sociétés", 3 : 97-102.

Deschodt L., Salvador P.G., Feray P., Schwenninger J.-L., 2012. Transect partiel de la plaine de la Scarpe (bassin de l'Escaut, Nord de la France). Stratigraphie et évolution paléo- géographique du Pléniglaciaire supérieur à l'Holocène récent. Quaternaire, 23, (1) : 87-116.

Dotterweich M., 2008. The history of soil erosion and fluvial deposits in small catchments of Central Europe : deciphering the long-term interaction between human and the environment - A review. Geomorphology, on line, [http:// www.101016/j.geomorph.200805023], consulté le 28/11/2013.

Dotterweich M., Dreibrodt S., 2011. Past land use and soil erosion processes in central Europe. Pages news, 19, (2) : 49-51.

Doyen E., Vannière B., Bichet V., Gauthier E., Richard H., Peтiт C., 2013. Vegetation history and landscape management from 6500 to 1500 cal.B.P. at Lac d'Antre, Gallo-Roman sanctuary of Villards d'Héria, Jura, France. Vegetation History and Archaeobotany, 22 : 83-97.

Duchaufour P., 1976. Atlas écologique des sols du monde, Masson. Ertlen D., Gebhardt A., Schneider N., Durand F., Thomas Y., Michler M., Schneikert F., Boës E., Schwartz D., 2013. Anthropisation et érosion agraire dans un paysage loessique (Bas-Rhin, France). In F. Bertoncello and J.-F. Berger (dir.), Variabilités environnementales, mutations sociales : nature, intensités, échelles et temporalités des changements. Actes des XXXII ${ }^{\mathrm{e}}$ Rencontres Internationales d'Archéologie et d'Histoire d'Antibes, 20-22 octobre 2011, sous presse.

Etienne D., Ruffaldi P., Ritz F., Dambrine E., 2010. Étude des variations de la végétation d'un massif forestier de la plaine lorraine (Moselle, France) depuis le Moyen Âge. Quaternaire, 21, (1) : 317-323.

Etienne D., Ruffaldi P., Goepp S., Ritz F., Georges-Leroy M., Pollier B., Dambrine E., 2011. The origin of closed depressions in Northeastern France: a new assessment. Geomorphology, 126 : 121-131.

Etienne D., Ruffaldi P., Dupouey J.-L., Georges-Leroy M., Ritz F., Dambrine E., 2013. Searching for ancient forests: A 2000 year history of land use in northeastern French forest deduced from the pollen compositions of closed depressions. The Holocene, 23, (5) : 678-691.

Fechner K., 1993. Ay-sur-Moselle, Rapport pédologique de la fouille de 1992. Rapport des Sites archéologiques de Gavisse-RD1 et Ay-sur-Moselle. Service régionale de l'Archéologie de Lorraine.

Fechner K., Langohr R., 1994. Résultats et problématique de l'étude pédologique de trois sites néolithiques en bordure de Moselle. Notae Praehistoricae, 13 : 115-117.

Fechner K., Langohr R., Mikkelsen J.H., Becze-Deak J., 1995. Affectation humaine et fertilité des sols au Néolithique ancien sur quelques sites du Grand-Duché de Luxembourg et de Lorraine. In C. Jeunesse (éd.). Le Néolithique danubien et ses marges entre Rhin et Seine. Actes du $22^{\mathrm{e}}$ colloque interrégional sur le Néolithique, Strasbourg 27-29 octobre 1995, Supplément aux cahiers de l'APRAA, 197-212. 
Fechner K., 2009. Rapport d'analyse pédologique. In F. Gérard (dir.). Vitry-sur-Orne, ZAC de la Plaine (57), Moselle, Tranches 1, 2, 3 et 4, nº $5772424 \mathrm{AH}$, Évolution d'un terroir du Néolithique Ancien au Xv siècle. Rapport Final d'Opération, INRAP-GEN, SRA Lorraine, Études paléo-environnementales, 4.

Fechner K., Slachmuylder J.-L., 2009. Les sols noirs anthropiques sur le tracé TGV Est : compréhension pédologique et malacologique, approche statistique et essai d'interprétation archéologique. In J. Vanmoerkerke (dir.). Le Bassin de la Vesle du Bronze Final au Moyen Âge à travers les fouilles du TGV Est. Bulletin de la Société Archéologique champenoise, 102, (2) : 335-361.

Fechner K., Baes R., Louwagie G., Gebhardt A., avec la collaboration de Deschodt L., Bécu B., Schartz E., 2014. Relic holocene buried colluvial and alluvial deposition in the basins of the Scheldt, the Meuse, the Seine and the Rhine (Belgium, Luxembourg and Northern France). A prospective state of research in rescue excavations. In E. Meylemans, J. Poesen, I. In't Ven (ed.). The Archaeology of Erosion, the Erosion of archaeology. Conference Brussels, 28-30 avril 2008. Relicta Monographien 9 (VIOE, Brussels) : 147-190.

Flageollet J.-C., 2002. Sur les traces des glaciers vosgiens. CNRS Éditions.

Franc O., 2005. Reconstitution paléo-environnementale à partir du contexte géomorphologique de quatre sites archéologiques de la plaine de l'est Lyonnais (Rhône, France). Quaternaire, 16, (2) : 95-105.

GARnier E., 2004. Terre de conquête, la forêt vosgienne sous l'ancien Régime. Fayard.

Gauthier E., Richard H., Ruffaldi P., 2000. Première tentative de reconstitution du couvert végétal de l'Est de la France à l'Âge du Fer. In T. Janin (ed.). Mailhac et le Premier Âge du Fer en Europe occidentale. Monographies d'Archéologie Méditerranéenne, 2000/7 : 195-204.

Gebhardt A, 1993. Soil micromorphological evidence of soil deterioration since the Mid-Holocene in Brittany, France. The Holocene, 3 : 333-341.

Gebhardt A., 1995. Soil micromorphological data from experimental and traditional agriculture. In Barham, Macphail (eds.). Archaeological sediments and soils: analysis, interpretation and management. Archetype Press, London, 5-40.

Gebhardt A., 1999. Micromorphological analysis of soil structural modification caused by different cultivation implements. In P. Anderson (ed.). Prehistory of agriculture, new experimental and ethnographic approaches. Monographie du CRA, 40, Institute of archaeology, University of California, Los Angeles, 260-266.

GebHardt A., 2000. Anthropisation, agricultures anciennes et formation des paysages ruraux : le rôle de la micromorphologie des sols, Études rurales, 153/154: 139-149.
Gebhardt A., 2003. Étude géomorphologique. In J. Millereux-Le Béchennec (dir.), Bliesbruck, Im Sand, RD82, rapport d'évaluation archéologique (1/08-5/08/2003). Bliesbruck-Metz, CG Moselle, SRA Lorraine.

Gebhardt A., 2004a. Lecture du sol : les archives de l'archéopédologue. La Gazette Lorraine, 56 : 27-28.

Gebhardt A., 2004b, Compte rendu géomorphologique. In X. Antoine (dir.). Illange-Bertrange (57, Moselle) « Mégazone». Rapport de diagnostic, INRAP-GEN, DRAC Lorraine.

Gebhardt A., 2004c. Rapport d'analyse micromorphologique. In L. Thomashausen (dir.). Illange (57, Moselle). Rapport Final d'Opération INRAP-GEN, SRA Lorraine.

Gebhardt A., 2004d. Rapport d'analyse micromorphologique. In L. Thomashausen (dir.). Ay-Sur-Moselle (57, Moselle). Rapport Final d'Opération INRAP-GEN, SRA Lorraine.

Gebhardt A., 2004e. Rapport d'analyse micromorphologique. In Gérard F. (dir.) Vitry-sur-Orne (57, Moselle). Rapport Final d'Opération INRAP-GEN, SRA Lorraine.

Gebhardt A., 2005. Rapport d'analyse micromorphologique. In A. Masquillier et M. Prévost (dir.). Solgne Eval-149 (57, Moselle). Rapport Final d'Opération INRAP-GEN, SRA Lorraine.

Gebhardt A, 2006. Étude géomorphologique. In R. Jude (dir.), Remomeix, "Les Mennes Hières" Phase1. Rapport de Diagnostic, INRAP-GEN, SRA Lorraine.

Gebhardt A., 2007a. Impact anthropique anciens sur les sols forestiers. Quelques études de cas en contexte archéologique et expérimental. In J.-L. Dupouey, E. Dambrine, C. Dardignac, and M. Georges-Leroy (éds.). La mémoire des forêts, Colloque : Forêt, archéologie et environnement 14-16 décembre 2004, INRA Nancy.

Gebhardt A., 2007b. Rapport d'analyse micromorphologique. In M. Gazenbeck (dir.). Metz-Borny (57, Moselle) "ZAC Sébastopol, secteur 5, Projet MAB”, Rapport Final d'Opération INRAP-GEN, SRA Lorraine.

Gebhardt A., 2008a. La pédologie d'un billon. In J. Burnouf (dir.). Archéologie médiévale en France, le second Moyen Âge (XIIXVI siècle). La Découverte, 43.

Gebhardt A., 2008b. Rapport d'analyse micromorphologique. In F. Thieriot (dir.). Ennery les Soldes. Rapport Final d'Opération, INRAP-GEN, SRA.

Gebhardt A., 2009. Rapport d'analyse micromorphologique. In F. Gérard (dir.). Vitry-sur-Orne, ZAC de la Plaine (57), Moselle, Tranches 1, 2, 3 et 4, n 5772424 AH, Evolution d'un terroir du Néolithique Ancien au Xv ${ }^{\mathrm{e}}$ siècle. Rapport Final d'Opération, INRAP-GEN, SRA Lorraine, Etudes paléo-environnementales, 4.

Gebhardt A., 2010a. Rapport d'analyse micromorphologique. In J. Millereux-Le Béchennec (dir.). Bliesbruck, Hinterer Sand, Im Sand, RD82, Rapport final d'opération de fouille préven- 
tive (04/04-18/08/2004). Bliesbruck-Metz-Saint-Denis, CG Moselle, SRA Lorraine.

Gebhardt A., 2010b. Rapport d'analyses micromorphologiques. In J.-D. Laffitte (dir.), Moyenvic (Moselle) "Les Crôleurs ", Contournement est D 955 (1999)/Rétablissement RD 914 (2001). Rapport d'opération d'archéologie préventive Afan/ lnrap, 3.

Gebhardt A., 2011a. Rapport d'analyse micromorphologique. In N. Meyer (dir.). Sarrebourg (54, Meurthe et Moselle), Rue du Sauvage. Rapport Final d'Opération INRAP-GEN, SRA.

Gebhardt A., 2011b. Rapport d'analyse micromorphologique. In F. Schembri (dir.). Lesmesnils "Chêne Brûlé » (57, Moselle), TGV-EST, site $n^{\circ} 188$, lot 34. DFS fouilles INRAPGEN, SRA.

Gebhardt A., 2011c, Rapport d'analyse micromorphologique. In S. Deffressigne (dir.). Bouxières-sous-Froidemont, Meurtheet-Moselle, (Le Tremble - La Tuilerie) : Un micro terroir de la fin de l'âge du Bronze à la période antique. Rapport Final d'Opération, INRAP-GEN, SRA Lorraine, Annexes et inventaires, 2.

Gebhardt A., 2011d. Rapport d'analyse micromorphologique. In P. Lefranc (dir.). Mittelhausen Kellen, Bas-Rhin, LGV EE, site 9-4. Rapport Final d'Opération, Fouille Archéologique, INRAP-GES, SRA Alsace.

Gebhardt A., 2014. Rapport d'analyse micromorphologique. In J.-M. Blaising (dir.). Rocade Sud de Metz (Section RD999-A31) Peltre (57) Val Saint Pierre site 5753425AH Site d'habitat de la protohistoire au VIII siècle, évolution du paysage de l'antiquité tardive au XXe s. (Afan 2000). Rapport final d'opération, INRAPGEN, SRA.

Gebhardt A., Georges-Leroy M., Rohmer P., Triboulot B., 2009. Apport de la micromorphologie des sols à l'interprétation de trois séquences sédimentaires lorraines bien datées. Archives pédologiques : pédoarchéologie et dynamique des paysages. Actes des $10^{\text {es }}$ Journées d'Étude des Sols, Strasbourg, 11-15/5/08. Session 1, 39-40.

Georges-Leroy M., Bock J., Dambrine E., Dupouey J.-L., Gebhardt A., Laffite J.-D., 2009. Un paysage agraire galloromain conservé dans le massif forestier de Haye (Meurthe-etMoselle). Actes de la table-ronde de Caen "Des hommes aux champs" octobre 2007, 157-180.

Germain-Vallée C., Lespez L., 2011. L'apport des recherches géomorphologiques et micromorphologiques récentes à l'archéologie des paysages de la plaine de Caen (Calvados, BasseNormandie). Norois, 220, (3) : 143-178.

Goepp S., Schwartz D., Thinon M., 2008. Influences anthropiques sur la végétation des Hautes-Vosges depuis le début de l'Holocène : apports de l'analyse pédo-anthracologique. In D. Galop (dir.). Paysages et Environnement. De la reconstitution du passé aux modèles prospectifs. Besançon, PU-FC, Annales
Littéraires. Série "Environnement, sociétés et archéologie", 177-184.

Guillet B., 1972. Relation entre l'histoire de la végétation et la podzolisation dans les Vosges, Thèse de doctorat de l'université de Nancy.

Gury M., 1973. Carte pédologique du plateau de Haye, Carte et Notice explicative, Centre de pédologie-Biologie, université Nancy 1, CNRS éd.

Gury M., 1976. Évolution des sols en milieu acide et hydromorphes sur terrasses alluviales de la Meurthe, Thèse de l'Université de Nancyl.

Holzhauser H., Magny M., Zumbuhl H., 2005. Glacier and lake level variations in west-Central Europe over the last 3500 years. The Holocene, 15, (6) : 789-801.

JaCQUin F., Florentin L., 1988. Atlas des sols de Lorraine, PU-Nancy.

Jude R., Gebhardt A., Wietold J., 2012. Haut-Clocher, Moselle, Ersterbach, LGV Est, Site 22, Anthropisation et gestion d'un vallon, de l'Antiquité au premier Moyen Âge. Rapport de fouille archéologique, Inrap GEN, Metz.

Kalis A.J., Knaap van der W.O., Schweitzer-Ralf A., 2006. A three thousand year succession of plant communities on a valley bottom in the Vosges Mountains, NE France, reconstructed from fossil pollen, plant macrofossils, and modern phytosociological communities. Vegetation History and Archaeobotany, $15: 377-390$.

Koenig M.-P., Ruffaldi P., 2007. Les habitats du bronze moyen en lorraine : approche culturelle, chronologique et spatiale. In H. Richard, M. Magny, C. Mordant (éd.). Environnement et cultures à l'Âge du Bronze en Europe occidentale, ACNSHS, 129 (DP21), CTHS, 159-178.

Kulinicz E., 1997. Géoarchéologie des paysages de la vallée de la Blies entre Bliesbruck et Reinheim. Mémoire de maîtrise, Département de géographie, Université de Nancy 2.

Kuzucuoglu C., Lespez L., Pastre J.-F., 1992. Holocene colluvial deposits on the slopes of the Paris Basin. In M. Bell and J. Boardman (eds), Past and present soil erosion, Archaeological and geographical perspectives. Oxbow monographes, 22 : 115-124.

LANGOHR R., 2001. L'anthropisation du paysage pédologique agricole de la Belgique depuis le Néolithique ancien - apports de l'archéo-pédologie. Étude et Gestion des sols, 8, (2) : 103-118.

Leopold M., Völkel J., 2006. Colluvium, definition, differentiation, and possible suitability for reconstructing Holocene climate data. Quaternary International, online, de [http:// www.10.1016/j.quaint.2006.10.030], consulté le 28/11/2013.

LE Roux J., 2007. Introduction à la géologie de l'Alsace-Lorraine et des régions limitrophes, In S. Cordier, D. Harmand, S. Occhietti (dir.). Livret guide de l'excursion AFEQ des 7-9 juin 2007, Lorraine, Luxembourg, Rhénanie-Palatinat, Sarre, 27-34.

ArcheoSciences, revue d'archéométrie, 38, 2014, p. 7-29 
Leroy M., Mangin M., Laurent H., Boukezzoula M., Raïssouni B., 2000. La sidérurgie dans l'est de la Gaule. L'organisation spatiale de la production de l'Âge du Fer au haut Moyen Âge. Gallia, 57 : 11-21.

Lespez L., 2012. Le temps des paysages, de l'environnement et des plaines littorales bas-normandes. In L. Lespez (éd.). Paysages et gestion de l'eau. Bibliothèque du Pôle Rural, 3 : 199-234.

LeVeau P., 2007, Archéologie et géoarchéologie d'une période historique dans les Alpes : les paradigmes épistémomogiques de la romanisation, Géomorphologie : reliefs, processus, environnement, $1: 85-98$.

Lexa-Chomard A., Pautrot C., 2006. Géologie et Géographie de la Lorraine. Éditions Serpenoises.

LuCAs-Leclin O., 1992. Étude géomorphologique de la grèzière de Domrémy-la-Pucelle (Vosges). Mémoire de maîtrise, Laboratoire de géographie physique, université de Nancy 2.

Macphail R.I., Courty M.-A., Gebhardt A., 1990. Soil micromorphological evidence of early agriculture in north-west Europe. World Archaeology, 22, (1) : 53-69.

Magny M., 1995. Une histoire du climat, des derniers mammouths au siècle de l'automobile. Errance.

Magny M., 2012. "Back to the futur" dans les Alpes? Une mise en perspective du réchauffement climatique en cours dans le contexte général de l'Holocène, sur la base de découvertes archéologiques. In J.-F. Berger (éd.). Des climats et des hommes. La Découverte.

Malrain F., 2000, "Fonctionnement et hiérarchies des fermes dans la société gauloise du III siècle à la période romaine : l'apport des sites de la moyenne vallée de l'Oise ", Thèse de l'Université de Paris 1-Panthéon-Sorbonne.

Mangerud J., Andersen S.T., Berglund B.E. Donner J.J., 1974, Quaternary stratigraphy of Norden, a proposal for terminology and classification. Boreas, 3 : 109-128

Mangerud J., Berglund B.E., 1978, The subdivision of the Quaternary of Norden : a discussion. Boreas, 7 : 178-181.

Mayewsin P. A., Rohling E., Stager J. C., Karlén W., Maasch K. A., Meekere L. A., Meyerson E. A., Gasse F., van Kreveld S., Holmgren K., Lee-Thorp J., Rosqvist G., Rack F., Staubwasser, M., Schneider R. R., Steig E. J., 2004. Holocene climate variability. Quaternary Research, 62 : 243-255.

Nilles R., Schneider N., Cicutta H., Gebhardt A., Wiethold J., 2010. Strasbourg, Bas-Rhin, 1 rue Sainte Marguerite, extension des bâtiments de l'ENA, Nouvelles découvertes en l'île Verte à Strasbourg : paléo-chenaux de la fin de l'âge du Bronze et occupation de La Tène finale. Rapport final de fouilles Archéologique, INRAP, GES, SRA Alsace.

Pastre J.-F., Leroyer C., Limondin-Lozouet N., Fontugne M., Hattié C., Krier V., Kunsech S., Saad M.-C., 2002. L'Holocène du Bassin Parisien : variations environnementales et réponses géo-écologiques des fonds de vallées. In $\mathrm{H}$. Richard, A. Vignot (éd.), Équilibres et ruptures dans les écosystèmes durant les 20 derniers millénaires en Europe de l'Ouest. Besançon, PU-FC, 831, Annales littéraires 730. Série "Environnement et sociétés", $3: 61-74$.

Petit C., Camerlynck C., Deweirdt E., Durlet C., Garcia J.-P. Gauthier E., Ollive V., Richard H., Wahlen P., 2006. Géoarchéologie du Site Antique de Molesme en vallée de Laigne (Côte-d'Or). Gallia, 63 : 263-281.

Prat B., 2009. Rapport d'analyse palynologique. In F. Gérard (dir.). Vitry-sur-Orne, ZAC de la Plaine (57), Moselle, Tranches 1, 2, 3 et 4, n 5772424 AH, Évolution d'un terroir du Néolithique Ancien au Xv ${ }^{\mathrm{e}}$ siècle, Rapport Final d'Opération, INRAP-GEN, SRA Lorraine, Études paléo-environnementales, 4.

Richard H., Ruffaldi P., 2004. Premières traces polliniques d'influence de l'homme sur le couvert végétal de l'Est de la France. In Néolithisation précoce. Premières traces d'anthropisation du couvert végétal à partir des données polliniques. Richard H. (dir.). Besançon, Presses Universitaires Franc-Comtoises, 2004, Annales Littéraires 777. Série « Environnement, sociétés et archéologie », $7:$ 117-126.

Ruffaldi P., 1999. Premières traces polliniques de néolithisation des zones de basse altitude de Lorraine (France). Quaternaire, $10,(4): 263-270$.

Ruffaldi P., Ritz F., Richard H., Dambrine E., Dupouey J.-L., 2006. Analyse pollinique de la mardelle d'Assenoncourt (Moselle, France) : impact des pratiques agricoles sur la biodiversité végétale en milieu forestier. Actes du colloque Forêt, archéologie et environnement, La mémoire des forêts, 69-77.

Salvador P.-G., Vérot-Bourrély A., Bravard, J.-P., Franc O., Macé S., 2002, Les crues du Rhône à l'époque gallo-romaine dans la région lyonnaise. In J.-P. Bravard, M. Magny (dir.), Les fleuves ont une histoire, paléo-environnement des rivières et des lacs français depuis 15000 ans. Archéologie aujourd'hui, Errance, 215-222.

Sell Y., Berchtold J.-P., Callot H., Hoff M., Gall J.-C., Walter J.-M., 1998. L'Alsace et les Vosges. La bibliothèque du naturaliste, Delachaux et Niestlé.

Sernander R., 1908. On the evidence of post-glacial changes of climate furnished by the peat mosses of northern Europe. Geologiska Föreningens i Stockholm Förhaldlinger, 30 : 365-478.

Sordoillet D., JoAn L., Girardclos O., 2013. La basse vallée de l'Ognon (Doubs et Jura, France) de l'Antiquité au Moyen Âge : Dynamique sédimentaire et dynamique du peuplement, in F. Bertoncello et J.-F. Berger (dir.). Variabilités environnementales, mutations sociales: nature, intensités, échelles et temporalités des changements. Actes des XXXII ${ }^{\mathrm{e}}$ rencontres Internationales d'Archéologie et d'Histoire d'Antibes, 20-22 octobre 2011. 
STOops G., 1996. Multilingual translation of the terminology used in the Handbook for Soil thin Section Description. Pedology, $36: 337-348$.

Stuiver M., Reimer P. J., 1993, Extended 14C database and revised CALIB radiocarbon calibration program. Radiocarbon, 35 : $215-230$.

Thiemeyer H, Dieter Blümel W., Dambeck R., Dieckmann B., Eberle J., Glade T., Hecht S., Houben P., Moldenhauer K.M., Schrott L., Schulte A., Vogt R., Wunderlich J., 2005. Soils, Sediments and slope process and their effects on sediment fluxes into the river Rhine. Erdkunde, Archiv für Wissenschaftiche Geographie, 59, Begründet von Carl Troll, BOSS, 184-198.

VAN Geel B., 2012. La crise climatique de 850 avant notre ère. In H. Richard, A. Vignot (éd.). Equilibres et ruptures dans les écosystèmes depuis 20000 ans en Europe de l'Ouest. Actes du Colloque de Besançon, septembre 2000.

Van Geel B., Magny M., 2002. Mise en évidence d'un forçage solaire du climat à partir de données paléo-écologiques et archéologiques : la transition Sub-boréal-Subatlantique. In H. Richard, A. Vignot (éd.). Équilibres et ruptures dans les écosystèmes depuis 20000 ans en Europe de l'Ouest. Actes du Colloque de Besançon, septembre 2000.

Vergne V., 2004. Les premiers indices d'impact de l'homme sur le couvert végétal des Vosges. Apport de la bibliographie. In H. Richard, (dir.). Néolithisation précoce. Premières traces d'anthropisation du couvert végétal à partir des données polliniques. Besançon, PU-FC, Annales Littéraires 777, Série "Environnement, sociétés et archéologie", 7 : 127-133.

Wahl L., 2007. Le climat de la Lorraine. In S. Cordier, D. Harmand, S. Occhietti (dir.). Livret guide de l'excursion AFEQ des 7-9 juin 2007, Lorraine, Luxembourg, RhénaniePalatinat, Sarre.

Wanner H., Ritz S. A web-based Holocene Climate Atlas, Université de Berne. [http://www.sritz.ch/resources/Research/ HOCLAT.pdf].

Wanner H., Solomina O., Grosjean M., Ritz S. P., Jetel M., 2011. Structure and origin of Holocene cold events. Quaternary Science Reviews, 30 : 3109-3123.

Weisrock A., Franoux D., 1993. Lenvironnement du site de Bliesbrück-Reinheim : Étude du fond de vallée de la Blies, Études offertes à J. Schaub, Blesa1, Editions Serpenoise. 